\title{
Data segmentation algorithms: Univariate mean change and beyond
}

\author{
Haeran $\mathrm{Cho}^{1}$ and Claudia Kirch ${ }^{2}$
}

July 9,2021

\begin{abstract}
Data segmentation a.k.a. multiple change point analysis has received considerable attention due to its importance in time series analysis and signal processing, with applications in a variety of fields including natural and social sciences, medicine, engineering and finance.

The goal of this survey article is twofold: In the first part, we review the existing literature on the canonical data segmentation problem which aims at detecting and localising multiple change points in the mean of univariate time series. We provide an overview of popular methodologies on their computational complexity and theoretical properties. In particular, our theoretical discussion focuses on the separation rate relating to which change points are detectable by a given procedure, and the localisation rate quantifying the precision of corresponding change point estimators, and we distinguish between whether a homogeneous or multiscale viewpoint has been adopted in their derivation. We further highlight that the latter viewpoint provides the most general setting for investigating the optimality of data segmentation algorithms.

Arguably, the canonical segmentation problem has been the most popular framework to propose new data segmentation algorithms and study their efficiency in the last decades. In the second part of this survey, we motivate the importance of attaining an in-depth understanding of strengths and weaknesses of methodologies for the change point problem in a simpler, univariate setting, as a stepping stone for the development of methodologies for more complex problems. We illustrate this with a range of examples showcasing the connections between complex distributional changes and those in the mean. We also discuss extensions towards high-dimensional change point problems where we demonstrate that the challenges arising from high dimensionality are orthogonal to those in dealing with multiple change points.
\end{abstract}

\footnotetext{
${ }^{1}$ School of Mathematics, University of Bristol, UK. Email: haeran.cho@bristol.ac.uk. Supported by the Leverhulme Trust Research Project Grant (RPG-2019-390).

${ }^{2}$ Department of Mathematics, Otto-von-Guericke University; Center for Behavioral Brain Sciences (CBBS); Magdeburg, Germany. Email: claudia.kirch@ovgu.de.
} 


\section{Introduction}

Change point analysis has a long tradition in statistics dating back to 1950s (see Page [148]) and has been a very active field of research ever since due to its importance in time series analysis, signal processing and many other applications where data is routinely collected over time in naturally nonstationary environments. This popularity can partly be explained by the fact that the assumption of piecewise stationarity underlying change point analysis, is one of the simplest forms of departure from stationarity while at the same time, it is found to be reasonable for many applications. There are also other types of non-stationarities such as unit roots or local stationarity that have been studied extensively in the literature which, however, will not be discussed in this article.

Research in change point analysis has predominantly focused on the following two directions: One line of research is on the development of sequential or online methodologies based on optimal stopping (see e.g. the recent books by Tartakovsky et al. [172] and Tartakovsky [171]) which is being actively studied to date but will not be part of this review. The second direction addresses the problem of change point detection in a posteriori or offline settings. Much of the research in the last century dealt with change point testing against the at-most-one-change (AMOC) alternative (see e.g. the books by Csörgö and Horváth [51] and Chen and Gupta [38]). Based on such tests, the location of a single change point can be estimated with the optimal localisation properties, typically by scanning for the point where the corresponding statistic attains its maximum. More recent papers tackle change point problems in complex situations (see the review papers by Aue and Horváth [15] and Horváth and Rice [89] and also Section 4 below), both sequentially and retrospectively, and this review paper focuses on the latter.

In retrospective change point analysis, it is often more realistic to allow for multiple change points, where the goal is to estimate both the total number and locations of the change points, a.k.a. data segmentation. While there already exist early papers showing the consistency of various change point procedures that make use of e.g. binary segmentation (i.e. iteratively testing for a change point and partitioning the data into two, [182]), or information criteria [125, 190], the problem of detecting multiple change points in data sequences experienced renewed interest in the statistical literature during the last 10 years. This was driven by several factors including the emergence of scientific problems requiring high-precision and high-speed segmentation algorithms: Such examples can be found in genomics [35, 128, 144, 145], astronomy [71], neurophysiology [140], climatology [156], cyber-security [1] and finance [41, to name but a few. Accordingly, there has been a surge of interest for computationally fast and statistically efficient methods for data segmentation, and most effort in this direction has been devoted to the problem of detecting multiple change points in the mean of univariate data. In this article, we also take this as an important starting point for developing change point methodologies in more complex situations. We first review the methods for detection 
and localisation of multiple change points in the mean of univariate data, which we refer to as the canonical segmentation problem, complementing the articles reviewing and comparing different change point detection algorithms such as [2, 61, 68, 178].

As noted by Brodsky and Darkhovsky [31] (see also the discussion in Section 4.1 below), more complex change point problems that allow changes in stochastic properties other than the mean, can be reduced to the (possibly multivariate) canonical segmentation problem after applying a suitable transformation to the input data that reveals the changes as those in the mean of the transformed data. Also, the challenges due to high dimensionality of the data, which is another important area of active research (see Section 4.2 below), are orthogonal to those arising from the presence of multiple change points over time. Thus we argue that the suite of algorithms developed for the canonical segmentation problem serve as a foundation for developing methodologies for more complex change point problems.

The rest of the paper is organised as follows. Section 2 describes the canonical segmentation problem including its aim and theoretical benchmarks, and Section 3 reviews the state-ofthe-art methodologies developed for this purpose. Section 4 provides an overview of the existing literature on more complex change point problems beyond mean changes and finite dimensionality, and discuss how the data segmentation algorithms described in Section 3 can be adapted for such problems.

\section{Multiple change point detection in the mean of univariate data}

We regard the following change point model

$$
X_{t}=f_{t}+\varepsilon_{t}=f_{0}+\sum_{j=1}^{q_{n}} d_{j} \cdot \mathbb{I}_{t \geq \theta_{j}+1}+\varepsilon_{t}, \quad \mathrm{E}\left(\varepsilon_{t}\right)=0 \text { and } \operatorname{Var}\left(\varepsilon_{t}\right)=\sigma^{2}, \quad t=1, \ldots, n,
$$

as the canonical change point model, where we denote by $q_{n}$ the total number of change points in the mean of $\left\{X_{t}\right\}_{t=1}^{n}$, by $\theta_{j}=\theta_{j, n}, j=1, \ldots, q_{n}$, their locations, by $d_{j}=d_{j, n}$ the (signed) magnitude of change and by $\delta_{j}=\delta_{j, n}=\min \left(\theta_{j}-\theta_{j-1}, \theta_{j+1}-\theta_{j}\right)$ the minimum distance from $\theta_{j}$ to its neighbouring change points, with the notational convention that $\theta_{0}=0$ and $\theta_{q_{n}+1}=n$. In data segmentation, the goal is to consistently estimate the number and the locations of the change points, and we review the methodologies proposed for this purpose in Section 3 ,

In contrast, in the corresponding testing problems, the aim is to test $H_{0}: q_{n}=0$ against $H_{1}: q_{n} \geq 1$; in the AMOC setting, the alternative hypothesis is given by $H_{1}: q_{n}=1$. There exist a wide variety of procedures proposed for change point testing, not only against the alternative of mean shifts but also allowing for more complicated structural breaks (see Section 4.1 for such examples). There are also a few methods that sequentially test for an 
increasing number of change points, i.e. $H_{0}: q_{n}=m$ against $H_{1}: q_{n}=m+1$ for given $m$ [21, 136]. These tests can be used as a pre-processing step to determine whether the data is sufficiently close to stationary, or whether a multiple change point detection methodology is required to segment the data into (approximately) stationary stretches. They can also serve as a basis for developing a data segmentation methodology, with the simplest example being the binary segmentation algorithm, see Section 3.2 below.

We assume that $\max _{1 \leq j \leq q_{n}}\left|d_{j}\right|=O(1)$ as well as $\min _{1 \leq j \leq q_{n}} \delta_{j} \rightarrow \infty$, separating the change point detection problem under (1) from that of outlier detection. Indeed, one of the main differences between the two problems is that in outlier detection, an increase in sample size does not benefit the performance of an outlier detection procedure beyond that it helps getting more precise estimators for the quantiles and, even so, the size of the outlier is required to be larger than these quantiles for its detection. On the other hand, in change point detection, the increased sample size (which corresponds to $\delta_{j} \rightarrow \infty$ under (1D) allows for the detection of local changes where $d_{j} \rightarrow 0$; therefore, looking for outliers in the differenced time series $\left\{X_{t}-X_{t-1}\right\}_{t=2}^{n}$ cannot provide an adequate solution to the change point problem.

Generally, the detectability of change points is determined by an interplay between the magnitude of changes and their distance to neighbouring change points: Large changes are detectable even if they are close to the adjacent change points while for smaller changes, they should be sufficiently distanced away from the neighbouring change points to be detectable. When large frequent changes and small changes over long stretches are simultaneously present in the same time series, it calls for a multiscale methodology that adapts to such situations and detects all change points. This gives rise to the distinction between a homogeneous change point scenario where the magnitude of changes and the spacing between change points are on the same scale for all change points, and the multiscale scenario where this is not the case. The difficulty of the canonical segmentation problem for a given time series under (1), is gauged by the detection lower bound $\Delta_{n}$ relating the magnitude of the change $d_{j}$ to the minimum distance to neighbouring change points $\delta_{j}$, and different formulations of the detection lower bound reflect these different scenarios, see Definition 2.1 (a) for the precise statement. When $\Delta_{n}$ diverges faster than the separation rate associated with a given methodology, changes are detectable with asymptotic power one and their locations can be estimated with accuracy.

Frequently, a methodology is deemed consistent in multiple change point detection with the localisation rate $\rho_{n}$, when it returns $\widehat{\Theta}=\left\{\widehat{\theta}_{j}, 1 \leq j \leq \widehat{q}: \widehat{\theta}_{1}<\ldots<\widehat{\theta}_{\widehat{q}}\right\}$ satisfying

$$
\widehat{q}=q_{n} \quad \text { and } \quad \max _{1 \leq j \leq q_{n}} w_{j}\left|\widehat{\theta}_{j} \cdot \mathbb{I}_{j \leq q_{n}}-\theta_{j}\right| \leq \rho_{n}
$$

with probability tending to one, for some $\rho_{n} / n \rightarrow 0$. The quantity $w_{j}$ denotes the difficulty associated with localising each change point $\theta_{j}$, which can also be formulated within the homogeneous or multiscale scenarios.

Definition 2.1, taken from Cho and Kirch [45, distinguishes multiscale formulations of detec- 
tion lower bound and localisation rate from their non-multiscale, homogeneous counterparts.

Definition 2.1. (a) Detection lower bound:

(i) Homogeneous change points: $\Delta_{n}=\min _{1 \leq j \leq q_{n}} d_{j}^{2} \cdot \min _{1 \leq j \leq q_{n}} \delta_{j}$.

(ii) Finite mixture of homogeneous change points: There are $N<\infty$ disjoint subsets of change points with their indices given by $\mathcal{J}_{k}, k=1, \ldots, N$, such that $\bigcup_{k=1}^{N} \mathcal{J}_{k}=\left\{1, \ldots, q_{n}\right\}$, whereby change points within each subset are homogeneous as defined in (i) and $\Delta_{n}=\min _{1 \leq k \leq N}\left(\min _{j \in \mathcal{J}_{k}} d_{j}^{2} \cdot \min _{j \in \mathcal{J}_{k}} \delta_{j}\right)$. A special case is when there are finitely many changes with $N=q_{n}$.

(iii) Multiscale change points: $\Delta_{n}=\min _{1 \leq j \leq q_{n}} d_{j}^{2} \delta_{j}$.

(b) Localisation rate: We distinguish between a homogeneous localisation rate where the estimation error for the $j$-th change point, $\left|\widehat{\theta}_{j}-\theta_{j}\right|$, is weighted globally with $w_{j}=$ $\min _{1 \leq j \leq q_{n}} d_{j}^{2}$, and a multiscale localisation rate where it is weighted locally with $w_{j}=d_{j}^{2}$.

In the AMOC situation where $q_{n} \leq 1$, all scenarios in Definition 2.1 (a) coincide and reduce to the assumption $d_{1}^{2} \min \left(\theta_{1}, n-\theta_{1}\right) \rightarrow \infty$ which is commonly found in the change point testing literature, see Section 1.5 of Csörgö and Horváth [51]. However, when $q_{n}>1$ (and possibly diverging with $n$ ), they truly reflect different scenarios: Proceeding from (i) to (iii) enlarges the associated parameter space, and thus (iii) provides the most general setting in which to investigate the detection power of a segmentation procedure. While most of the literature deals with the homogeneous setting (see Section 3.3), it is often too restrictive and does not permit large frequent jumps alternate with small jumps over long segments - a situation naturally embedded in the multiscale setting. In Definition 2.1 (b), the multiscale localisation rate relates the difficulty in accurate localisation of each change point to the size of the corresponding jump.

Propositions 2.1 and 2.2 state the benchmarks for the minimax optimal separation and localisation rates, which have been established under the special case where $\left\{\varepsilon_{t}\right\}_{t=1}^{n}$ are i.i.d. (sub-)Gaussian random variables. To the best of our knowledge, there do not exist equivalent results on the detection lower bound or the localisation rate (when $q_{n} \rightarrow \infty$ ) beyond the case of i.i.d. sub-Gaussianity. For the AMOC alternative, it is well known that the minimax optimal localisation rate is given by $O_{P}(1)$ (see Korostelev [119]), i.e. $\rho_{n} \rightarrow \infty$ arbitrarily slowly in (2). This rate extends beyond Gaussianity and independence when there are a finite number of change points, see Corollary 4.4 of Cho and Kirch [45].

Proposition 2.1 (Proposition 1 of Arias-Castro et al. [10], lower bound on the minimax separation rate). Under (1), let $H_{0, n}: q_{n}=0$ and $H_{1, n}$ describe the setting where $q_{n}=2, d_{n}:=d_{1}=-d_{2}$ and $\delta_{n}:=\theta_{2}-\theta_{1}$ with $n^{-1} \delta_{n} \rightarrow 0$. Then, $H_{0, n}$ and $H_{1, n}$ are asymptotically inseparable if $\left|d_{n}\right| \sqrt{\delta_{n}} \leq \sqrt{2 \log \left(n / \delta_{n}\right)}-\nu_{n}$ where $\nu_{n} \rightarrow \infty$. 
Proposition 2.2 (Proposition 6 of Fromont et al. [73], lower bound on the minimax localisation rate for possibly an unbounded number of change points). Under (1) with $q_{n} \geq 2$, let $\left|d_{j}\right|=: d_{n}$ for all $j=1, \ldots, q_{n}$, and denote by

$$
\begin{array}{r}
\Xi=\left\{\left(\theta_{1}, \ldots, \theta_{q_{n}}\right): 0 \equiv \theta_{0}<\theta_{1}<\ldots<\theta_{q_{n}}<\theta_{q_{n}+1} \equiv n, \quad\right. \text { and } \\
\left.d_{n}^{2} \min _{1 \leq j \leq q_{n}}\left(\theta_{j+1}-\theta_{j-1}\right)>c \log \left(q_{n}\right)\right\}
\end{array}
$$

for some $c>0$, the parameter space for the locations of change points. Then, for some $C>0$,

$$
\inf _{\mathcal{K} \in \mathbb{N}^{q_{n}}} \sup _{\Theta \in \Xi} \mathrm{E}_{\Theta}\left\{d_{H}(\mathcal{K}, \Theta)\right\} \geq C d_{n}^{-2} \log \left(q_{n}\right)
$$

where $d_{H}(\mathcal{K}, \Theta)=\max \left\{\max _{k \in \mathcal{K}} \min _{\theta \in \Theta}|k-\theta|, \max _{\theta \in \Theta} \min _{k \in \mathcal{K}}|\theta-k|\right\}$ denotes the Hausdorff distance.

\section{Review of data segmentation methodologies}

Broadly, algorithms for the canonical segmentation problem can be categorised into two: One line of research casts the problem as that of globally optimising an objective function, which imposes a penalty on the model complexity given e.g. by the number of change points; the other is closely related to the change point testing literature, whereby the test for detecting a single change point is applied locally for detection and estimation of multiple change points. We briefly mention that there exist methods based on hidden Markov models where the main focus lies in modelling such phenomena, with algorithms for estimating the sequence of hidden states [79, 177]. There are also methods allowing for Bayesian inference under change point models [25, 60, 66, 159, 189, see Eckley et al. [61] for further references.

In what follows, we review the literature on the canonical segmentation problem under (1) according to the above categorisation, providing brief descriptions of representative methodologies and their computational complexity as well as theoretical properties in relation to the benchmark provided in Section 2.

\subsection{Global optimisation methods}

\subsection{1 $\ell_{0}$-penalisation}

Information criteria have popularly been adopted in change point problems. Under (1), the Schwarz criterion [164, SC] takes the form

$$
\mathrm{SC}(\mathcal{A})=\frac{n}{2} \log \left(\frac{\operatorname{RSS}(\mathcal{A})}{n}\right)+p_{n}(\mathcal{A}) \quad \text { where }
$$




$$
\operatorname{RSS}(\mathcal{A})=\sum_{j=0}^{m} \sum_{t=k_{j}+1}^{k_{j+1}}\left(X_{t}-\bar{X}_{k_{j}: k_{j+1}}\right)^{2} \quad \text { with } \quad \bar{X}_{a: b}=\frac{1}{b-a} \sum_{t=a+1}^{b} X_{t} .
$$

The function $p_{n}(\mathcal{A})$ denotes the penalty imposed on the complexity of a model determined by a set of change point candidates $\mathcal{A}=\left\{k_{j}, 1 \leq j \leq m: k_{1}<\ldots<k_{m}\right\} \subset \mathcal{I}_{1}:=\{1, \ldots, n-1\}$ (with $k_{0}=0$ and $k_{m+1}=n$ ); a popular choice is $p_{n}(\mathcal{A})=\lambda_{n}|\mathcal{A}|$ where $\lambda_{n}$ is a tuning parameter. For some integer $N \leq n$, we denote a collection of all subsets of $\mathcal{I}_{1}$ with their cardinality bounded by $N$, by $\mathfrak{A}[N]=\left\{\mathcal{A} \subset \mathcal{I}_{1}:|\mathcal{A}| \leq N\right\}$. Then, minimising $\operatorname{SC}(\mathcal{A})$ over $\mathcal{A} \in \mathfrak{A}[N]$ returns a set of change point estimators, $\widehat{\Theta}(\mathfrak{A}[N])=\min _{\mathcal{A} \in \mathfrak{A}(N)} \operatorname{SC}(\mathcal{A})$.

With the penalty linear in the number of candidate change points as $p_{n}(\mathcal{A})=(1+\epsilon) \log (n) \cdot|\mathcal{A}|$, the consistency of $\mathrm{SC}$ in estimating the total number of change points has been established under i.i.d. Gaussianity, in Yao [190] (with $\epsilon=0$ ) and Lee [125] (with a small constant $\epsilon>0$ ), in the setting where both $q_{n}$ and $N$ are fixed with $N>q_{n}$. This result is complemented by Yao and $\mathrm{Au}$ [192], where the consistency of the least squares estimators of $\theta_{j}, 1 \leq j \leq q_{n}$, is established and their distribution is derived when $q_{n}$ is fixed and known. Penalties depending on the spacing between the change points as well as the total number $|\mathcal{A}|$, are considered in Chen et al. [37, Pan and Chen [149] and Zhang and Siegmund [194], and the application of the Akaike information criterion to the change point problem is studied in Ninomiya [143]. Boysen et al. [30] consider the minimisation of the Potts functional

$$
\widehat{\Theta}(\mathfrak{A})=\arg \min _{\mathcal{A} \in \mathfrak{A}} P_{0}(\mathcal{A}), \quad \text { where } \quad P_{0}(\mathcal{A})=\frac{1}{n} \operatorname{RSS}(\mathcal{A})+p_{n}(\mathcal{A})
$$

with $p_{n}(\mathcal{A})=\lambda_{n}|\mathcal{A}|$, over $\mathcal{A} \in \mathfrak{A}[n-1]=$ : $\mathfrak{A}$, i.e. without any bound on the cardinality of $\mathcal{A}$ other than the trivial one. Under i.i.d. sub-Gaussianity, they show the consistency of $\widehat{\Theta}(\mathfrak{A})$ both in estimating the total number and the locations of change points, provided that $\lambda_{n} \rightarrow 0$ and $n \lambda_{n} / \log (n) \rightarrow \infty$. Lavielle and Moulines [123] establish the asymptotic consistency of the minimiser of the Potts functional under general conditions on $\left\{\varepsilon_{t}\right\}$, given that a suitable upper bound on the number of change points $N$ is available. Wang et al. [185] extend the results of Boysen et al. [30] to the settings where $q_{n} \rightarrow \infty, \min _{1 \leq j \leq q_{n}}\left|d_{j}\right| \rightarrow 0$ and $n^{-1} \min _{1 \leq j \leq q_{n}} \delta_{j} \rightarrow 0$ as $n \rightarrow \infty$, with the penalty $p_{n}(\mathcal{A})=\log ^{1+\epsilon}(n) \cdot|\mathcal{A}|$ for some small $\epsilon>0$. By considering a location-dependent penalty $p_{n}(\mathcal{A})=n^{-1} L\left\{\lambda|\mathcal{A}|+2 \sum_{j=0}^{m} \log \left(n /\left(k_{j+1}-k_{j}\right)\right)\right\}$ with some tuning parameters $L, \lambda>0$, Fromont et al. 73 . show that the minimisation of $P_{0}(\mathcal{A})$ achieves minimax optimality in both detection lower bound and change point localisation, matching the rates provided in Propositions 2.1 2.2 .

There exist dynamic programming algorithms which solve the $\ell_{0}$-penalised least squares estimation problem in (5). The segment neighbourhood approach [19] requires an upper bound on the number of change points, say $N$, and solves the constrained minimisation problems for a varying number of change points, attaining the computational complexity of $O\left(N n^{2}\right)$. The optimal partitioning [105] approach, by recursively searching for the optimal partition 
conditioning on the latest change point estimator, achieves the computational complexity of $O\left(n^{2}\right)$ for a given choice of the penalty. There are several methods which aim at speeding up the dynamic programming algorithms by pruning the search space, such as the pruned exact linear time method [110], the functional pruning technique [157] and their extensions [135]. Haynes et al. 85] provide a methodology for finding the solution path for a range of penalty parameters $\lambda \in\left[\lambda_{\min }, \lambda_{\max }\right]$, when the penalty increases linearly in the size of $\mathcal{A}$ as $p_{n}(\mathcal{A})=\lambda|\mathcal{A}|$, while Lebarbier [124] proposes the slope heuristic method that examines how the segmentation result varies with such $\lambda$. Tickle et al. 176 investigate the parallelisation of dynamic programming-based methods for fast computation when $n$ is very large. Imposing an autoregressive (AR) structure of order one on $\left\{\varepsilon_{t}\right\}$, Chakar et al. 34, propose an adaptation of (3) and provide an algorithm for approximating its global minimiser. Further allowing for random walk-like fluctuations, Romano et al. [162 provides an efficient dynamic programming algorithm that returns an exact solution of the minimisation problem.

Finally, we mention that the objective function based on the minimum description length principle [83, 161] imposes an $\ell_{0}$-type penalty on the model complexity, and has successfully been applied to piecewise parametric modelling [17, 52, 53 in combination with the genetic algorithm to traverse the parameter space and solve the optimisation problem.

\subsection{2 $\ell_{1}$-penalisation}

While efficient algorithms exist for the optimisation of $\ell_{0}$-penalised objective functions, the computational complexity typically scales quadratically in $n$. Relaxing the $\ell_{0}$-penalty in (5) to an $\ell_{1}$-penalty (a.k.a. total variation penalty) gives rise to

$$
P_{1}(\mathbf{g})=\frac{1}{n} \sum_{t=1}^{n}\left(X_{t}-g_{t}\right)^{2}+\lambda_{n} \sum_{t=1}^{n-1}\left|g_{t+1}-g_{t}\right|, \quad \mathbf{g}=\left(g_{1}, \ldots, g_{n}\right)^{\top},
$$

which is closely related to the fused Lasso, originally introduced in Tibshirani et al. [174 to take into account the ordering of the features in the data. The locations of change points are estimated by the locations of jumps in $\widehat{\mathrm{g}}=\arg \min _{\mathbf{g} \in \mathbb{R}^{n}} P_{1}(\mathrm{~g})$. Harchaoui and LévyLeduc [84] show that adopting the least angle regression proposed by Efron et al. [62] in the context of $\ell_{1}$-penalised linear regression, the computational complexity of solving for $\widehat{\mathrm{g}}$ is $O\left(N^{3}+N n \log (n)\right)$, where $N$ denotes the maximal number of change point candidates to be considered and needs to be specified. The properties of the $\ell_{1}$-penalised least squares estimator $\widehat{\mathrm{g}}$, both in its $\ell_{2}$-error in estimating the mean and change point localisation, has been studied in Rinaldo [160] and Harchaoui and Lévy-Leduc 84] under i.i.d. (sub-)Gaussianity. Lin et al. [130] provide a near-minimax optimal rate $($ up to $\log \log (n))$ for the $\ell_{2}$-error when the change points are sparse, but also show that $\widehat{\mathbf{g}}$ tends to over-estimate the number of change points and requires further post-processing for consistent estimation of $q_{n}$. 


\subsubsection{Multiscale change point segmentation methods}

Li et al. [129] define a class of multiscale change point segmentation (MCPS) methods, which include the simultaneous multiscale change point estimators controlling for the family-wise error rate [72, SMUCE] and the false discovery rate [128, FDRseg] in estimating the number of change points. They solve a (non-convex) optimisation problem, which searches for the most parsimonious candidate estimator of the piecewise constant function over the acceptance region of a suitable multiscale test. More specifically, the optimisation problem is given by

$$
\min _{g \in S[0,1)} \# J(g) \quad \text { subject to } \max _{\substack{I \in \mathcal{M} \\ g \equiv \gamma_{I} \text { on } I}}\left\{\frac{1}{\sqrt{|I|}}\left|\sum_{t / n \in I}\left(X_{t}-\gamma_{I}\right)\right|-s_{I}\right\} \leq \eta,
$$

where $\# J(g)$ denotes the number of jumps in $g \in S[0,1)$, the space of right-continuous step functions on $[0,1)$ (whose change points lie on the grid $\{i / n\}_{i=0}^{n-1}$ ), $\mathcal{M}$ a multiscale system of sub-intervals of $[0,1)$ fulfilling a set of requirements, $s_{I}$ a scale penalty and $\eta$ a threshold controlling the over-estimation probability.

Frick et al. [72] establish the near-minimax optimality of SMUCE as given in Propositions 2.1 2.2 , under the exponential family regression model with the parameter varying over time in a piecewise constant manner, and discuss an efficient implementation of the optimisation problem in (7) via dynamic programming. For the canonical segmentation problem, Pein et al. [150] and Dette et al. [58] address the issues arising from heteroscedasticity and serial dependence in $\left\{\varepsilon_{t}\right\}_{t=1}^{n}$, respectively.

\subsubsection{Model selection-based segmentation}

Arlot and Celisse [11] investigate the use of cross-validation for the change point problem under (1). With $\operatorname{RSS}(\mathcal{A})$ defined in (4) serving as the empirical risk measure of a change point model $\mathcal{A}$, the proposed method adopts the leave-one-out or $K$-fold cross-validation methods for final model selection, in place of imposing a penalty on the model complexity as the class of methods discussed in Sections 3.1.1 and 3.1.2. The paper provides a dynamic programming approach to efficiently compute the procedure and studies its theoretical performance in terms of the $\ell_{2}$-error.

\subsection{Local testing methods}

The methodologies belonging to this class generally proceed in two stages: First, the data is scanned for candidate change point estimators, from which the final estimators are selected via a model selection method.

In the first step, candidate generation is achieved by multiscale scanning of the data for change points using the following weighted cumulative sum (CUSUM) statistics computed on 
the segment $\left\{X_{t}, s<t \leq e\right\}$ :

$$
\mathcal{T}_{s, k, e}(X)=\sqrt{\frac{(k-s)(e-k)}{e-s}}\left(\bar{X}_{s: k}-\bar{X}_{k: e}\right) \quad \text { where } \quad \bar{X}_{a: b}=\frac{1}{b-a} \sum_{t=a+1}^{b} X_{t},
$$

for $0 \leq s<k<e \leq n$. In the AMOC setting, it is well-known that the location of the maximum of the CUSUM statistics given by $k_{\circ}=\arg \max _{0<k<n}\left|\mathcal{T}_{0, k, n}(X)\right|$, is a consistent minimax optimal estimator for the change point. This motivates taking the maximiser of the CUSUM statistics as a change point candidate within the local interval $(s, e)$ provided that $\max _{s<k<e}\left|\mathcal{T}_{s, k, e}(X)\right|$ is large enough.

A variety of methods adopt $\mathcal{T}_{s, k, e}(X)$ in order to scan the data for change points with a method-specific search space $\mathcal{S} \subset \mathcal{I}_{3}:=\{(s, k, e): 0 \leq s<k \leq n\}$, which is either fixed, random or data-dependent. The construction of the search space aims at isolating change points for their detection and localisation: Ideally, for each change point, $\mathcal{S}$ contains at least one interval which is as large as possible for the detection of even a small change, while containing only a single change point for its localisation and thus avoiding the contamination by multiple change points (further discussion on this point is found in Section 3.2.1). Once candidate change point estimators are identified, it remains to determine the final set of estimators which is achieved by a model selection (pruning) methodology, such as thresholding or information criteria.

In Sections 3.2.1 3.2.3, we discuss candidate generation strategies which scan the data using $\mathcal{T}_{s, k, e}(X)$ with different search spaces $(s, k, e) \in \mathcal{S}$. Then in Section 3.2.4, we describe popular model selection methodologies applied to the thus-generated candidate change point estimators.

\subsubsection{Binary segmentation algorithm and its extensions}

Binary segmentation (BS) algorithm [165, 182] is a generic method for multiple change point estimation which recursively partitions the data into two. For multiple change point detection under (1), it identifies a candidate estimator $k_{1}$ from scanning the CUSUM statistic $\left|\mathcal{T}_{0, k, n}(X)\right|, 1 \leq k<n$, as its maximiser. If the maximum CUSUM (max-CUSUM) statistic $\mathcal{T}_{0, n}^{\max }=\max _{1 \leq k<n}\left|\mathcal{T}_{0, k, n}(X)\right|$ is sufficiently large, the data is partitioned into $\left\{X_{t}\right\}_{t=1}^{k_{1}}$ and $\left\{X_{t}\right\}_{t=k_{1}+1}^{n}$, and the scanning and maximisation of the CUSUM statistics is repeated over each partition separately until a stopping criterion is met. The search space of BS is stochastic, as the search space at the $i$-th iteration with $i \geq 2$ depends on the change points detected at earlier iterations, and its complexity is typically $O(n \log (n))$. Venkatraman [179] established the consistency of BS while allowing the number of change points, $q_{n}$, to diverge with $n$.

The CUSUM statistic coincides with the likelihood ratio statistic for testing the null hypothesis of no change point $\left(H_{0}: q_{n}=0\right)$ against the AMOC alternative $\left(H_{1}: q_{n}=1\right)$ under i.i.d. Gaussian errors, and thus is particularly well-adopted for the detection and localisation 

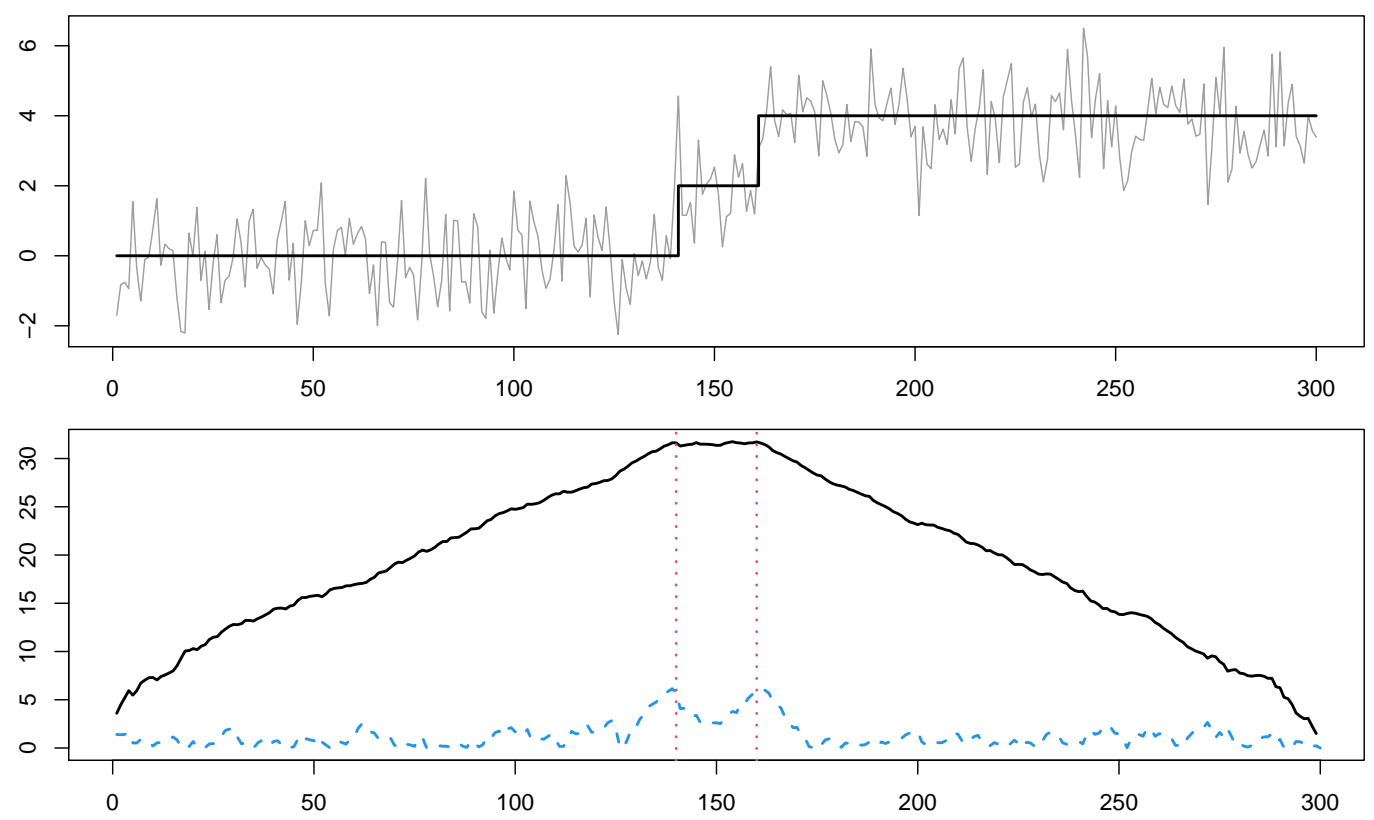

Figure 1: Top: A realisation $\left\{X_{t}\right\}_{t=1}^{n}$ from (1) with $f_{t}=2 \cdot \mathbb{I}_{t \geq 141}+2 \cdot \mathbb{I}_{t \geq 161}, \varepsilon_{t} \sim_{\text {iid }} \mathcal{N}(0,1)$ and $n=300$ and the piecewise constant signal $f_{t}$ (bold). Bottom: $\left|\mathcal{T}_{0, k, n}(X)\right|, 1 \leq k<n$ (solid), and $\left|\mathcal{T}_{k-G, k, k+G}(X)\right|, k \in \mathcal{S}_{\mathrm{M}}(G)$ with a boundary correction and $G=15$ (broken, see Section 3.2 .2 for further details), plotted with the vertical dotted lines denoting the locations of $\theta_{j}, j=1,2$.

of a single change point. On the other hand, in the presence of multiple change points, BS often performs poorly in estimating the locations of the change points as the search space may include intervals contaminated by more than one change points. The CUSUM-based test still attains good power when applied to such intervals, but the statistic may be unsuitable for locating the change points due to this contamination. Figure 1 provides an illustration where, while $\left|\mathcal{T}_{0, k, n}(X)\right|, 1 \leq k<n$, attain large values near the two change points, their precise locations are not well pointed out by the statistic. In such a situation, procedures that aim at isolating each change point for its detection and localisation, such as the moving sum procedure (see Section 3.2.2), perform better in locating the change points although they tend to lack power by comparison when used solely for testing.

The wild binary segmentation (WBS) algorithm proposed in Fryzlewicz [74] sets out to remedy the limitation of BS by scanning the data over a random grid $\mathcal{R}_{n} \subset \mathcal{I}_{2}:=\{(s, e): 0 \leq s<$ $e \leq n\}$, which amounts to examining $\mathcal{T}_{s, k, e}(X),(s, k, e) \in \mathcal{S}_{\mathrm{W}}\left(\mathcal{R}_{n}\right)$ with

$$
\mathcal{S}_{\mathrm{W}}\left(\mathcal{R}_{n}\right)=\left\{(s, k, e): k=s+1, \ldots, e,(s, e) \in \mathcal{R}_{n}\right\}
$$

As in BS, WBS recursively identifies candidate change point estimators and partitions the data into two, but the search is performed over the randomly drawn background intervals and thus its computational complexity is determined by the choice of $\mathcal{R}_{n}$ as $O\left(n\left|\mathcal{R}_{n}\right|\right)$. In order 
to ensure that each change point is considered on its own within a large enough interval for its detection and estimation, WBS requires that a sufficiently large number of background intervals are drawn; more precisely, $\left|\mathcal{R}_{n}\right|$ is required to increase as the minimum distance between the change points, $\delta_{n}:=\min _{1 \leq j \leq q_{n}} \delta_{j}$, decreases such that $\left(n / \delta_{n}\right)^{-2}\left|\mathcal{R}_{n}\right| \rightarrow \infty$. Fryzlewicz [74] refines the theoretical analysis of Venkatraman [179], and shows that WBS outperforms BS in terms of both the separation and the localisation rates.

There exist several modifications and extensions of WBS. Wang et al. [185] consider WBS with the lengths of the background intervals in $\mathcal{R}_{n}$ bounded from above by $\delta_{n}$, which ensures that each background interval contains a single change point at most, leading to an improvement of the localisation rate. Baranowski et al. 23. propose a change point-like feature detection method termed the narrowest-over-threshold (NOT) where, at each recursive iteration, the shortest interval $(s, e]$ over which the associated max-CUSUM statistic, $\max _{s<k<e}\left|\mathcal{T}_{s, k, e}(X)\right|$, exceeds a given threshold is selected, rather than the interval returning the largest max-CUSUM. Fryzlewicz [76] proposes WBS2, which iteratively draws random intervals and produces a complete solution path, i.e. an ordered sequence of $\mathcal{I}_{1}=\{1, \ldots, n-1\}$ according to their importance measured by the associated CUSUM statistics. Kovács et al. [120] investigate the seeded binary segmentation (SBS), which generates the search grid $\mathcal{R}_{n}$ in a deterministic fashion. In Lu et al. [133, a sampling strategy is investigated in combination with BS and WBS for handling very large $n$.

In all above, the theoretical investigation is focused on the case of uncorrelated data, while Cho and Fryzlewicz [44] discuss the usefulness of the WBS-like strategy in separating the effects of change points and time series fluctuations in the presence of serial correlations. Finally, we mention the circular binary segmentation proposed in Venkatraman and Olshen [180] for the analysis of array CGH data, where the test statistics are tailored for detecting changes of epidemic nature, where $f_{\theta_{2 j+1}}=f_{\theta_{2 j+2}+1}$ for $j=0, \ldots,\left\lfloor q_{n} / 2\right\rfloor$ under (1).

\subsubsection{Moving sum procedures}

The moving sum (MOSUM) procedure has been adopted for simultaneously detecting and locating multiple change points [47, 63, 102]. For the canonical segmentation problem it scans the data for change points using the statistics $\mathcal{T}_{s, k, e}(X)$ over a fixed grid $(s, k, e) \in \mathcal{S}_{\mathrm{M}}(G)$, which is fully determined by the choice of a bandwidth $G=G_{n}$ as

$$
\mathcal{S}_{\mathrm{M}}(G)=\{(s, k, e): s=k-G, e=k+G, k=G, \ldots, n-G\} .
$$

The search space is of cardinality $\left|S_{\mathrm{M}}(G)\right|=O(n)$ since for every time point $k$, only the local interval $(s, e)=(k-G, k+G)$ is considered; we sometimes denote that $k \in \mathcal{S}_{\mathrm{M}}(G)$ where there is no confusion. Eichinger and Kirch 63 propose two criteria for the simultaneous localisation of the change points whereby each estimator is obtained as a local maximiser of $\left|\mathcal{T}_{s, k, e}(X)\right|, k \in \mathcal{S}_{\mathrm{M}}(G)$, over a sufficiently large interval relative to $G$, at which the MOSUM 
statistic exceeds a theoretically motivated threshold.

Messer et al. [140, 141] consider a multiscale extension of the MOSUM procedure with the search space

$$
\mathcal{S}_{\mathrm{M}}(\mathcal{G})=\left\{(s, k, e): s=k-G_{h}, e=k+G_{h}, k=G_{h}, \ldots, n-G_{h} ; h=1, \ldots, H\right\}
$$

where $\mathcal{G}=\left\{G_{1}, \ldots, G_{H}\right\}$ denotes a set of bandwidths. The screening and ranking procedure of Niu and Zhang [144] also adopt a multiscale MOSUM procedure for candidate generation. The use of asymmetric bandwidths $\mathbf{G}=\left(G_{\ell}, G_{r}\right)$ with $\mathcal{S}_{\mathrm{M}}(\mathbf{G})=\left\{(s, k, e): s=k-G_{\ell}, e=\right.$ $\left.k+G_{r}, k=G_{\ell}, \ldots, n-G_{r}\right\}$ has been investigated by Meier et al. [139]. Cho and Kirch [45] propose the following scheme for asymmetric bandwidth selection based on the Fibonacci series $\left\{F_{m}\right\}$ constructed as $F_{m}=F_{m-1}+F_{m-2}, m \geq 2$ with $F_{0}=F_{1}=1$ : The set of asymmetric bandwidths is determined as

$$
\mathcal{H}_{n}=\left\{\left(G_{\ell}, G_{r}\right): G_{\ell / r}=G_{\min } \cdot F_{\ell / r}, 1 \leq \ell, r \leq H_{n}, \quad \text { and } \quad \frac{\max \left(G_{\ell}, G_{r}\right)}{\min \left(G_{\ell}, G_{r}\right)} \leq C_{\text {asym }}\right\},
$$

with $H_{n}$ chosen such that $G_{H_{n}}<\lfloor n / \log (n)\rfloor$ for some minimum allowable bandwidth $G_{\text {min }}$ and a fixed constant $C_{\text {asym }} \geq 1$. The latter constant controls the unbalancedness of the asymmetric bandwidth $\left(G_{\ell}, G_{r}\right)$ such that the computational complexity of the multiscale MOSUM procedure involving $T_{s, k, e}(X),(s, k, e) \in \mathcal{S}_{\mathrm{M}}\left(\mathcal{H}_{n}\right)$ is $O(n \log (n))$.

The multiscale application of the MOSUM procedure necessitates a pruning procedure for the removal of conflicting estimators detected for the identical change points at multiple bandwidths, as well as possibly spurious estimators detected at small bandwidths.

\subsubsection{Bottom-up and sequential approaches}

There exist data segmentation methods that set out to isolate each change point by considering the data from finer to coarser scales. Fryzlewicz 75 propose a method based on the tailgreedy unbalanced Haar (TGUH) transform, which performs a bottom-up data decomposition using the unbalanced Haar wavelet basis. Starting from the finest grid $\mathcal{S}^{(0)}=\{1,2, \ldots, n-1\}$, it identifies the regions (defined by the adjacent elements of the grid) to be merged as where the corresponding statistics $\left|\mathcal{T}_{s, k, e}(X)\right|$ computed over $(s, k, e)=\left(k_{i-1}^{(0)}, k_{i}^{(0)}, k_{i+1}^{(0)}\right), k_{i}^{(0)} \in \mathcal{S}^{(0)}$ (with $k_{0}^{(\ell)}=0$ and $k_{\left|\mathcal{S}^{(\ell)}\right|+1}^{(\ell)}=n$ for all $\ell \geq 0$ ) are small, and accordingly update the search grid. Repeatedly applying the above steps until all $\left|\mathcal{T}_{s, k, e}(X)\right|$ are large enough over $(s, k, e)=$ $\left(k_{i-1}^{(\ell)}, k_{i}^{(\ell)}, k_{i+1}^{(\ell)}\right), k_{i}^{(\ell)} \in \mathcal{S}^{(\ell)}$ for some $\ell$, we obtain an ordering of change point candidates with an implicit tree-like structure on which model selection is performed. By greedily selecting the regions to be merged, which is managed by a parameter controlling their proportion out of the remaining regions, TGUH achieves a computational complexity of $O\left(n \log ^{2}(n)\right)$. A similar idea of bottom-up change point analysis has also been explored in the reverse segmentation proposed by Chan and Chen [35], and the backward procedure of Shin et al. [167]. 
The pseudo-sequential procedure of Venkatraman [179] adapts an online change point detection algorithm to the offline data segmentation problem. The Isolate-Detect (IDetect) proposed in Anastasiou and Fryzlewicz [4] operates in a similar vein, sequentially searching for change points from both ends. More specifically, it scans $\mathcal{T}_{s, k, e}(X),(s, k, e) \in \mathcal{S}_{L}(\ell, r) \cup$ $\mathcal{S}_{R}(\ell, r)$, where $\mathcal{S}_{L}(\ell, r)$ and $\mathcal{S}_{R}(\ell, r)$ denote the search spaces containing the intervals expanded from the left to the right, and those expanded from the right to the left, respectively, for given $0 \leq \ell<r \leq n$, as

$$
\begin{aligned}
& \mathcal{S}_{L}(\ell, r)=\left\{(s, k, e): \ell=s<k<e \leq r \text { with } e=m \lambda_{n}, m \in \mathbb{N}\right\}, \\
& \mathcal{S}_{R}(\ell, r)=\left\{(s, k, e): \ell \leq s<k<e=r \text { with } s=n-m \lambda_{n}, m \in \mathbb{N}\right\},
\end{aligned}
$$

where $\lambda_{n}$ denotes a parameter satisfying $\lambda_{n} \leq \delta_{n}=\min _{1 \leq j \leq q_{n}} \delta_{j}$. Starting with $(\ell, r)=(0, n)$, it iteratively updates $(\ell, r)$ with the previously detected change point estimators. The choice of $\lambda_{n}$ involves balancing between the computational complexity $O\left(n^{2} \lambda_{n}^{-1}\right)$, and the change point localisation property (smaller $\lambda_{n}$ providing better guarantee of isolating each change point for their detection and localisation).

\subsubsection{Model selection}

The methods outlined in Sections 3.2.1 3.2.3 produce a set of candidate change point estimators $\mathcal{C}$ from scanning the data at multiple scales; some return a subset of $\mathcal{I}_{1}=\{1, \ldots, n-1\}$, while the others generate a (complete) solution path ordering the elements of $\mathcal{I}_{1}$ according to their importance. For the estimation of the total number and the locations of the change points, it remains to select the final model from the set of candidate estimators.

Closely related to the change point testing literature, thresholding is popularly adopted for model selection in change point detection. Eichinger and Kirch [63] derive the (distributional) limit behaviour of the MOSUM test statistic $\max _{k \in \mathcal{S}_{\mathrm{M}}(G)}\left|\mathcal{T}_{s, k, e}(X)\right|$ under the null hypothesis of no change, under general conditions allowing for heavy tails and serial dependence in $\left\{\varepsilon_{t}\right\}$ with the bandwidth $G$ satisfying $G \rightarrow \infty$ while $G / n \rightarrow 0$; the result is extended for the asymmetric MOSUM statistic in Meier et al. [139]. Messer et al. [140, 141] approximate the MOSUM statistic by a Gaussian process when $G$ grows linearly with $n$. With such asymptotic null distributions at hand, we can derive a theoretically motivated threshold at a chosen significance level and control for the family-wise error rate.

For methods such as BS, WBS and their extensions, the test statistics associated with change point estimators are dependent on the previously detected ones, and thus it is challenging to investigate their asymptotic null distributions. Instead, the thresholds are often given by a range depending on $n$, the level of noise and other typically unknown quantities such as $\min _{j}\left|d_{j}\right|$ and $\min _{j} \delta_{j}$, see [4, 23, 74, 75, 179, 185]. Fang et al. 65] provide a detailed analysis of the tail probability of $\max _{(s, k, e) \in \mathcal{I}_{3}}\left|\mathcal{T}_{s, k, e}(X)\right|$ under i.i.d Gaussianity, which enables the 
selection of the threshold at a prescribed significance level.

For a critical value or a threshold to be useful, we need to estimate the level of noise given by the error variance $\sigma^{2}=\operatorname{Var}\left(\varepsilon_{t}\right)$ under independence or the long-run variance $\tau^{2}=\operatorname{Var}\left(\varepsilon_{t}\right)+$ $2 \sum_{h=1}^{\infty} \operatorname{Cov}\left(\varepsilon_{0}, \varepsilon_{h}\right)$ when serial dependence is permitted. Several estimators exist for both $\sigma^{2}$ and $\tau^{2}$ under the model (1) [20, 58, 173], but the difficulty associated with the estimation of the latter has been well-documented particularly in the presence of multiple change points [152], and the former also becomes challenging to estimate when frequent change points are present. As a remedy, Fryzlewicz [76] proposes the 'steepest drop to low levels' (SDLL) model selection methodology applicable with a complete solution path ordering $\mathcal{I}_{1}=\{1, \ldots, n-1\}$, with thresholding adopted as a secondary criterion.

Information criteria such as those discussed in Section 3.1.1, have frequently been adopted for final model selection in combination with the methods belonging to the local testing category. Fryzlewicz [74] propose to minimise the strengthened SC, defined in (3) with the penalty function $p_{n}(\mathcal{A})=\log ^{1+\epsilon}(n) \cdot|\mathcal{A}|$ for some small $\epsilon>0$, along a solution path $\left\{k_{1}\right\} \subset$ $\left\{k_{1}, k_{2}\right\} \subset \ldots \subset\left\{k_{1}, \ldots, k_{N}\right\} \subset \mathcal{I}_{1}$ returned by WBS with some pre-determined $N>q_{n}$; the subset that minimises the criterion is chosen as the final model. A similar approach is also taken by [4, 23, 144].

Alternatively, Cho and Kirch [45] propose a top-down pruning methodology based on SC, which searches for the final model $\widehat{\Theta}$ from a set of candidate change point estimators, say $\mathcal{C}$, according to the following rules: (i) Adding further estimator to $\widehat{\Theta}$ monotonically increases SC, and (ii) among all subsets satisfying (i), $\widehat{\Theta}$ attains the minimal cardinality and (if there exist ties) the minimum SC. This approach gains computationally compared to minimising the SC exhaustively among all the $2^{|\mathcal{C}|}$ subsets of $\mathcal{C}$, and achieves consistency in multiple change point estimation under general assumptions permitting dependence and heavy tails in $\left\{\varepsilon_{t}\right\}$. A localised version of the pruning method attaining linear complexity is proposed for further computational consideration, and it is shown to match the minimax optimal multiscale separation and localisation rates when applied to the candidates generated by a multiscale MOSUM procedure. Cho and Fryzlewicz [44] explore the top-down approach under a parametric modelling assumption on $\left\{\varepsilon_{t}\right\}$, which effectively avoids the difficult task of evaluating the information criterion at a model under-specifying the number of change points.

Messer et al. [140, 141] consider a bottom-up merging method for the multiscale MOSUM procedure: Accepting all the estimators from the smallest bandwidth, it proceeds to coarser scales and only accepts a change point estimator if its detection interval does not contain any estimators that are already accepted. A similar strategy, named the local segmentation, has also been considered in Chan and Chen [35, which permits asymmetric bandwidths. 
Table 1: Comparison of change point detection methodologies on their detection lower bound and the separation and localisation rates derived under i.i.d. (sub-)Gaussianity, where $d_{n}=$ $\min _{1 \leq j \leq q_{n}}\left|d_{j}\right|$ and $\delta_{n}=\min _{1 \leq j \leq q_{n}} \delta_{j}$. Also, we provide the computational complexity of different methods with $N$ denoting the maximum allowable number of change points where relevant, and list their software implementations. The results reported here for BS and WBS are taken from [43, 188.

\begin{tabular}{|c|c|c|c|c|c|c|c|}
\hline & \multicolumn{2}{|c|}{ Detection lower bound } & \multicolumn{2}{|r|}{ Localisation } & \multirow{2}{*}{$\begin{array}{l}\text { Computational } \\
\text { complexity }\end{array}$} & \multirow[b]{2}{*}{ Implementations } \\
\hline & & $\Delta_{n}$ & Separation rate & $w_{j}$ & Rate & & \\
\hline $\begin{array}{l}\ell_{0^{-}} \\
\text {penalisation }\end{array}$ & $\begin{array}{l}73 \\
185\end{array}$ & $\begin{array}{c}\min _{j} d_{j}^{2} \delta_{j} \\
d_{n}^{2} \delta_{n}\end{array}$ & $\begin{array}{c}\log \left(n / \delta_{n}\right) \\
\log (n)\end{array}$ & $\begin{array}{l}d_{j}^{2} \\
d_{j}^{2}\end{array}$ & $\begin{array}{c}\log \left(q_{n}\right) \\
\log (n)\end{array}$ & $\begin{array}{l}O\left(n^{2}\right) \\
O\left(n^{2}\right)\end{array}$ & 111. 49. 158] \\
\hline $\begin{array}{l}\ell_{1^{-}} \\
\text {penalisation }\end{array}$ & $\begin{array}{l}84 \\
130\end{array}$ & $\begin{array}{l}- \\
-\end{array}$ & $\begin{array}{l}- \\
-\end{array}$ & $\begin{array}{c}1 \\
d_{n}^{2}\end{array}$ & $\begin{array}{c}\log ^{2}(n) \\
\log (n) \log \log (n)\end{array}$ & $\begin{array}{c}O\left(N^{3}+\right. \\
N n \log (n))\end{array}$ & - \\
\hline MCPS & $\begin{array}{l}72 \\
129 \\
\end{array}$ & $\begin{array}{l}d_{n}^{2} \delta_{n} \\
d_{n}^{2} \delta_{n}\end{array}$ & $\begin{array}{c}\log \left(n / \delta_{n}\right) \\
q_{n} \log (n)\end{array}$ & $\begin{array}{l}d_{n}^{2} \\
d_{n}^{2} \\
\end{array}$ & $\begin{array}{c}\log (n) \\
q_{n} \log (n)\end{array}$ & $\begin{array}{c}O\left(n^{2}\right) \\
-\end{array}$ & 151. 127] \\
\hline $\mathrm{BS}$ & 74 & $d_{n}^{2} \delta_{n}$ & $n^{3} \delta_{n}^{-5 / 2} \log (n)$ & $d_{n}^{2}$ & $\left(n / \delta_{n}\right)^{5} \log (n)$ & $O(n \log (n))$ & 111 \\
\hline WBS & $\begin{array}{l}74 \\
185 \\
\end{array}$ & $\begin{array}{l}d_{n}^{2} \delta_{n} \\
d_{n}^{2} \delta_{n}\end{array}$ & $\begin{array}{c}\left(n / \delta_{n}\right)^{4} \log (n) \\
\log (n)\end{array}$ & $\begin{array}{l}d_{j}^{2} \\
d_{j}^{2}\end{array}$ & $\begin{array}{c}\left(n / \delta_{n}\right)^{4} \log (n) \\
\log (n)\end{array}$ & $\begin{array}{c}O\left(n\left|\mathcal{R}_{n}\right|\right) \\
\text { with }\end{array}$ & 5 \\
\hline NOT & 23 & $d_{n}^{2} \delta_{n}$ & $\log (n)$ & $d_{j}^{2}$ & $\log (n)$ & $\left(n / \delta_{n}\right)^{2} /\left|\mathcal{R}_{n}\right| \rightarrow 0$ & \\
\hline MOSUM & $\begin{array}{l}63 \\
45 \\
35 \\
\end{array}$ & $\begin{array}{c}d_{n}^{2} \delta_{n} \\
\min _{j} d_{j}^{2} \delta_{j} \\
d_{n}^{2} \delta_{n}\end{array}$ & $\begin{array}{c}\log \left(n / \delta_{n}\right) \\
\log (n) \\
\log \left(n / \delta_{n}\right)\end{array}$ & $\begin{array}{l}d_{j}^{2} \\
d_{j}^{2} \\
d_{j}^{2}\end{array}$ & $\begin{array}{l}\log \left(q_{n}\right) \\
\log \left(q_{n}\right) \\
\log (n)\end{array}$ & $\begin{array}{c}O(n) \\
O(n \log (n)) \\
O(n \log (n))\end{array}$ & 138 \\
\hline $\begin{array}{l}\text { IDetect } \\
\text { TGUH }\end{array}$ & $\begin{array}{l}4 \\
75 \\
\end{array}$ & $\begin{array}{l}d_{n}^{2} \delta_{n} \\
d_{n}^{2} \delta_{n}\end{array}$ & $\begin{array}{l}\log (n) \\
\log ^{2}(n)\end{array}$ & $\begin{array}{l}d_{j}^{2} \\
d_{n}^{2}\end{array}$ & $\begin{array}{l}\log (n) \\
\log ^{2}(n)\end{array}$ & $\begin{array}{c}O\left(n^{2} / \delta_{n}\right) \\
O\left(n \log ^{2}(n)\right)\end{array}$ & 5. 3] \\
\hline
\end{tabular}

\subsection{An overview of change point detection methodologies}

Table 1 provides a comprehensive overview of change point methodologies discussed in Sections 3.1 3.2, which expands upon Table 1 of Cho and Kirch [45]. They are compared on their theoretical properties such as how the detection lower bound is formulated, their separation and localisation rates under i.i.d. (sub)-Gaussianity (according to Definition 2.1 and (2)) and computational complexity, and their software implementations are listed where available.

\subsubsection{Numerical performance}

We briefly discuss the practical performance of the representative change point methods in Table 1, based on the numerical experiments conducted in [45] using their respective implementations listed in the table; see also Fearnhead and Rigaill [67] for an extensive comparison of data segmentation algorithms based on their numerical performance, and the vignette accompanying the $\mathrm{R}$ package breakfast [5] comparing various CUSUM-based candidate generation and model selection methods.

We emphasize that different algorithms excel at different performance criteria, and their performance vary with different test signals. For instance, dynamic programming algorithms for minimising the $\ell_{0}$-penalised cost functions, such as [110] and [135] (implemented in [111] and [158], respectively), take a fraction of a second in processing long data sequences $(n \geq$ $2 \times 10^{4}$ ), followed by the multiscale MOSUM procedure with bottom-up merging [35, 140]; the SDLL model selection method of [76] is well-suited to detect a large number of frequent 
change points; FDRseg [128] shows favourable performance for relatively short test signals; TGUH [75] tends to incur few false positives even when change points are sparse while $n$ is large. Between those methods that employ information criteria for model selection, the localised pruning proposed in [45] outperforms the sequential minimisation along the solution path considered in [74] in terms of correctly estimating the number of change points. Overall, the localised pruning methodology shows well-rounded performance in combination of either MOSUM- or CUSUM-based candidate generating procedures on a variety of test signals with both sparse and dense change points.

These observations apply to the case of i.i.d. Gaussian errors. Most of the methods considered above are not easily extended to cope with heavy-tailed or serially correlated $\left\{\varepsilon_{t}\right\}$, as their tuning parameters are specifically tailored for the i.i.d. (sub-)Gaussian setting. The localised pruning, on the other hand, provides a framework for selecting a theoretically-motivated penalty in the $\mathrm{SC}$ that is well-justified in the numerical experiments where $\left\{\varepsilon_{t}\right\}$ are generated as i.i.d. random variables following $t_{5}$-distributions, or $\operatorname{AR}(1)$ processes with both strong and weak autocorrelations.

\subsection{Inference for the multiple change point problem}

In this section, we review the literature on inference for multiple change points in the context of the canonical segmentation problem. For a review of the literature in the case of a single change point, see Jandhyala et al. [106].

In order to quantify the uncertainty in the detection of multiple change points, MCPS methods (see Section 3.1.3) such as SMUCE [72] and FDRseg [128] allow for controlling the probability of over-estimating the number of change points in terms of family-wise error rate or false discovery rate. Eichinger and Kirch [63] provide the asymptotic null distribution of the test statistic of the single-scale MOSUM procedure, namely that of $\max _{(s, k, e) \in \mathcal{S}_{\mathrm{M}}(G)}\left|\mathcal{T}_{s, k, e}(X)\right|$ (see (8)) when there is no change point present, under general assumptions permitting heavy tails and serial dependence in $\left\{\varepsilon_{t}\right\}$. Using this distribution, we can test whether or not there exists a mean shift within the $G$-environment at each time point $k$, where the thus-obtained $p$ value controls the family-wise error rate by construction. There also exist resampling methods for change point tests in the i.i.d. setting which generate better small sample approximations of the p-values, see Antoch and Hušková [8] and also Hušková [94 for a survey. For the case of i.i.d. Gaussian errors, Fang et al. [65] give the approximation of the tail probabil-

ity of $\max _{0 \leq s<k<e \leq n}\left|\mathcal{T}_{s, k, e}(X)\right|$ (maximum of the CUSUM statistics over all possible triplets $(s, k, e))$ under the null hypothesis of no change points, which in turn provides an approximation of the probability of a false positive error.

Post-selection inference methods exist for the change point problem when $\left\{\varepsilon_{t}\right\}$ are i.i.d. Gaussian, where the interest lies in testing for the equality of the mean around estimated change point locations conditional on their estimation process, see e.g. Hyun et al. [104] and Jew- 
ell et al. 107. Thanks to the conditioning sets being polyhedral in the case of BS and its variants, or via efficient characterisation of the conditioning set afforded by the functional pruning technique [135] for minimising the $\ell_{0}$-penalised objective functions (Section 3.1.1), the corresponding $p$-values can be computed from truncated normal distributions; however, their interpretation is involved and this approach requires further correction for multiple testing. Fryzlewicz [77] considers an alternative framework called post-inference selection under which the interest lies in identifying intervals containing at least one change points at a given global significance level without locating them.

In a more classical sense, there are also a few methods for quantifying the uncertainty about the locations of change points. Eichinger and Kirch 63] derive the asymptotic distribution of the MOSUM-based change point estimators in the case of local changes (i.e. $d_{j} \rightarrow 0$ ), and Cho and Kirch [46] show that the corresponding limit for the fixed change case (i.e. $d_{j}$ constant) depends on the unknown distribution of $\left\{\varepsilon_{t}\right\}$, extending similar observations made in the AMOC setting for CUSUM-based estimators [7, 9]. This motivates constructing bootstrap confidence intervals around change points, as proposed by [7, 9] in the case of i.i.d. errors and by [96, 97] in time series settings, all for the AMOC problem. Meier et al. [139] outline the bootstrap construction of pointwise and uniform confidence intervals for multiple change points based on the MOSUM procedure, and Cho and Kirch [46] show its theoretical validity for both local and fixed changes in the presence of i.i.d. (not necessarily Gaussian) $\left\{\varepsilon_{t}\right\}$. In addition, MCPS [72, 128 provide a confidence set for all candidate signals from which confidence intervals around the change points can be obtained. Using the inverse relation between confidence intervals and hypothesis tests, 65. describe how approximate confidence regions can be generated.

\section{Extensions to more complex problems}

In this section, we discuss several avenues for extending the methodologies from Section 3 to more complex change point problems beyond that of detecting change points in the mean of univariate data sequence under (1), which include high-dimensional change point analysis. While some literature in these directions already exists, it is far scarcer than the literature on the canonical data segmentation problem. We argue that a thorough understanding on theoretical and computational performance of different data segmentation methods for the simpler problem in the univariate setting, forms the basis for the methodological development in more complex situations.

In Section 4.1, we discuss that many tests for changes in the stochastic properties other than the mean (mostly in the AMOC scenario), are closely related to those proposed for detecting a change in the mean of univariate data. Therefore, data segmentation algorithms that have proven useful for the canonical segmentation problem can be adapted for complex change 
point detection problems in combination with such tests. These combined methodologies are expected to deliver satisfactory or even optimal performance, although they require more sophisticated mathematical analysis which in turn necessitates an in-depth understanding of both parts to be combined.

In Section 4.2 , we give a brief overview of the state-of-the-art methodologies for high-dimensional change point problems. Focusing on the problem of change point detection in the mean of high-dimensional panel data, we show that the challenges in dealing with high dimensionality are orthogonal to those arising from the presence of multiple change points.

\subsection{Extensions beyond detecting mean changes under Gaussianity}

First we provide a brief review of the existing literature on the detection of multiple change points in stochastic properties other than the mean, in univariate or multivariate data sequences. Under parametric models for linear and non-linear time series, $\ell_{0^{-}}$[17, 53, 54] and $\ell_{1}$-penalisation [26, 36] methods, the MOSUM procedure based on likelihood ratio statistic [193] and BS [78] have been adopted for detecting change points in the parameters that vary over time in a piecewise constant manner. Data segmentation under piecewise polynomial (mostly linear) models have been addressed in [23, 69, 134, 175], also adopting the algorithms discussed in Section 3. For nonparametric change point analysis, BS [137], WBS [147] and $\ell_{0}$-penalisation [12, 80, 196] have been combined with various methods for measuring distributional changes, and some computational efforts have been made to improve the dynamic programming algorithms for the methods in the latter category [33, 86]. In the time series setting, methods based on local periodograms [154] and wavelet transform [41] have been developed in combination with the MOSUM procedure and BS, respectively.

As seen in the above, data segmentation problems beyond that of mean change point detection require a methodology appropriate for revealing and measuring the time-varying characteristics of the data, which is of separate concern from handling multiple change points. We can tackle complex data segmentation problems by combining suitable methodologies addressing the two different aspects. This in turn requires an in-depth understanding of both strengths and weaknesses of the procedures for the canonical data segmentation problem as discussed in Section 3, in addition to understanding how extensions beyond detecting mean changes are achieved. We will use the remainder of this section to focus on the latter aspect and provide a short overview of the techniques developed mainly in the AMOC setting.

The effective dimensionality of data segmentation problems is given by the number of unknown parameters such that even for univariate observations, the effective dimensionality of a given problem can be large, see e.g. Kirch and Kamgaing [113] for an example for testing for parameter stability in non-linear autoregressive models by means of a semiparametric approach based on neural networks, which involves a large number of parameters. To make the problem even more difficult, statistics for revealing changes (e.g. obtained after a suitable 
data transformation) are often not independent even if the observed data is. Bücher et al. [32] consider a test for detecting changes in the serial dependence using a copula approach, and discuss how the result from such a test can be combined with those from change point tests for distributional changes (all based on resampling methodology) even though these tests are dependent. Kirch et al. [116] describe in detail how testing for changes in moderately large linear vector autoregressive processes becomes a high-dimensional problem, and provide discussions on how to resolve the complications arising from the high dimensionality. This shows that techniques for high-dimensional change point analysis as discussed later in Section 4.2 . are also of relevance for data segmentation under complex time series models.

\section{Data transformation}

Some complex change point problems can be reduced to that of detecting changes in the mean by adopting a suitable data transformation where typically, the dimensionality of the transformed data is larger (sometimes much larger) than that of the original observation sequence. For example, the problem of detecting changes in the covariance of $p$-dimensional data $\mathbf{X}_{t}=\left(X_{1 t}, \ldots, X_{p t}\right)^{\top}$ (under the assumption of a constant mean), can be transformed to that of detecting changes in the mean of $p(p+1) / 2$-dimensional data by considering the vector consisting of cross-products, i.e. $\mathbb{X}_{t}=\left(X_{i t} X_{j t}: 1 \leq i \leq j \leq p\right)^{\top}$. Clearly, the effective dimensionality is quadratic in the dimension of the original data. Also, the components of $\mathbb{X}_{t}$ are not independent and the dependence is not negligible. Such a transformation has been adopted for change point analysis in the covariance structure of multivariate [16], functional [170] and high-dimensional [169] data, and Cho and Fryzlewicz [42] propose a similarly motivated wavelet transform for the segmentation of high-dimensional time series in the second-order structure.

\section{Generalised method of moments}

The above approach is applicable only if the parameters of interest, which are subject to change over time, can be expressed as the expected values of an appropriately transformed data, i.e. as some moments. For most parametric models, however, this is not the case. For example, suppose that $\left\{X_{t}\right\}$ follows a (centered) linear AR model of order one, i.e.

$$
X_{t}=\alpha X_{t-1}+e_{t}, \quad e_{t} \sim \mathrm{WN}\left(0, \sigma^{2}\right), \quad t=1, \ldots, n .
$$

Then, the AR parameter $\alpha$ cannot be written as any moment after a simple data transformation. However, it can be written as a generalised moment, namely the quotient $\mathrm{E}\left(X_{t} X_{t-1}\right) / \mathrm{E}\left(X_{t}^{2}\right)$, as given by the well-known Yule-Walker equation and moreover, can be estimated (locally) from the data by using the method of moments. This observation gives rise to the following two approaches for change point analysis. 
The Wald approach compares directly the distance between the estimators obtained from different stretches of the data: At location $k$, we evaluate $d\left(\widehat{\beta}_{0: k}, \widehat{\beta}_{k: n}\right)$ where $d$ is some measure of distance, and $\widehat{\beta}_{a: b}$ denotes the estimator for the parameter of interest $\beta$ obtained from the data stretch $\left\{X_{t}, a<t \leq b\right\}$. The Wald approach leads to good performance if the parameters are identifiable and the local estimators can be obtained without resorting to numerical algorithms. On the other hand, its application may not be appropriate when there exist many (local) optima of the objective function for parameter estimation. Such a situation arises e.g. for neural networks, where different parameters describing the same or very similar regression functions might be far from each other in the parameter space. Further, this is particularly problematic if the estimators can only be obtained numerically. Even when the objective function does not have multiple local maxima, if it is relatively flat around the true parameter, it can be misleading to gauge the distance between the estimators of the parameters instead of the processes that they describe.

A score-type approach is more robust in this respect and is closely related to the CUSUM statistic described in Section 3.2. In contrast to simple data transformations as described previously, the transformations adopted in the score-type approach often involve the (local) parameter estimators. Let us illustrate this by re-visiting the example of a linear AR(1) time series $(9)$ in the AMOC situation, where the score-type statistic is given by

$$
\frac{1}{\sqrt{n}} \max _{2 \leq k<n}\left|\sum_{t=2}^{k} X_{t-1}\left(X_{t}-\widehat{\alpha}_{0: n} X_{t-1}\right)\right|
$$

where $\widehat{\alpha}_{0: n}$ denotes an estimator of the AR parameter based on the full data set. Some authors suggest to use partial sums of estimated residuals; however, the thus-constructed test has power only against some alternatives, namely those leading to changes in the mean of estimated residuals (see e.g. [116]). The statistic in (10) bears clear resemblance to the CUSUM statistic for mean change detection (written below with slightly different locationdependent weights from Section 3.2 for better comparison):

$$
\frac{1}{\sqrt{n}} \max _{1 \leq k<n}\left|\sum_{t=1}^{k}\left(X_{t}-\bar{X}_{0: n}\right)\right| .
$$

Here, $\bar{X}_{0: n}$ can be regarded as an estimator of the unknown mean based on the full sample, analogously to $\widehat{\alpha}_{0: n}$ being the global estimator of $\alpha$.

There are many other examples where the score-type statistics are adopted for change point analysis, including the tests based on likelihood ratio or classical $M$-estimators (see the next subsection on robust change point analysis) and those for changes in linear (auto)regression [103, 116], integer-valued time series such as Poisson autoregressive processes [112] and even regression models based on neural networks [113]. In all of the above, the test statistics are 
based on partial sums

$$
\frac{1}{\sqrt{n}} \max _{1 \leq k<n}\left\|\sum_{t=1}^{k} F\left(\mathbf{Y}_{t}, \widehat{\beta}_{0: n}\right)\right\| \quad \text { where } \sum_{t=1}^{n} F\left(\mathbf{Y}_{t}, \widehat{\beta}_{0: n}\right)=0,
$$

where $F$ is a suitable estimating function and $\widehat{\beta}_{0: n}$ the global estimator based on the full sample from this estimating function. The norm $\|\cdot\|$ often involves the unknown covariance matrix of $F\left(\mathbf{Y}_{t}, \beta\right)$ or an appropriate estimator thereof. In many instances, $\mathbf{Y}_{t}$ is not the original observation sequence; e.g. in the above $\operatorname{AR}(1)$ example, $\mathbf{Y}_{t}$ contains lagged observations. This shows that effectively, one is looking for mean changes in the pseudo-data $F\left(\mathbf{Y}_{t}, \widehat{\beta}_{0: n}\right)$, whose dimension is equal to the number of unknown parameters. For linear models, the Wald and the score-type statistics are equivalent in the AMOC setting, see Kirch and Kamgaing 112. In non-linear situations, this does not hold in small samples but does so asymptotically in well-behaved situations.

The Wald approach can be extended to multiple change point detection e.g. in combination with the MOSUM procedure by comparing the distance between local estimators over moving windows. Alternatively, the corresponding moving sum score statistic with a local estimator can be used. However, both suffer from the problem of having to calculate $O(n)$ local estimators which, as noted above, brings in further numerical challenges when there does not exist an analytic solution to the estimating function. Using a slightly modified score-type statistic replacing the local estimator with a global inspection parameter, say $\widetilde{\beta}$ (which may be data-dependent), alleviates this issue but typically, there is no guarantee that all changes are detectable as changes in the mean of $F\left(\mathbf{Y}_{t}, \widetilde{\beta}\right)$. Reckrühm [155] shows that even so, at least one change is made detectable therein, which naturally leads to adopting several of such inspection parameters. Some first results have been obtained by Reckrühm [155] and Kirch and Reckrühm [115, on the combined methodology of the MOSUM procedure and the use of estimating functions for the detection of multiple change points in general settings.

\section{Robust change point analysis}

The framework of generalised method of moments also prominently includes robust tests for changes in the mean or regression parameters (often known as $M$-estimation in this context). Selecting a suitable estimating function $F$ (related to what is known as a score function), one can obtain not only more robust estimators of the parameters of interest but also more robust methods to test for their changes. For example, under the mean change model as in (1) with $q_{n} \leq 1$, choosing $F(X, \beta)=\mathbb{I}_{\{X>\beta\}}-\mathbb{I}_{\{X<\beta\}}$ (with $\beta$ corresponding to $f_{0}=\mathrm{E}\left(X_{t}\right)$ when there exists no change point) results in $\widehat{\beta}_{0: n}$ that coincides with the median where $\sum_{t=1}^{n} F\left(X_{t}, \widehat{\beta}_{0: n}\right)=0$. Such an approach is also known as $L_{1}$-procedure and discussed e.g. in Hušková [91 for detecting changes in linear regression. Theoretical investigation into change point detection procedures based on $M$-estimation can be found in [6, 91, 92, 93, 101] under 
the i.i.d. assumption, and in [98, 153] for dependent data. A more detailed survey of the literature on this topic, including references on a-posteriori as well as sequential change point testing, is given in [95]. The paper includes some discussions on rank-based tests as well, which is an alternative approach to achieve robustness in change point testing. One such an example is the generalisation of the Wilcoxon rank sum test to the change point setting which is a special case of a (non-degenerate) $U$-statistic. $U$-statistics are adopted for the estimation of a range of different parameters far beyond the mean, and also used in testing for the stability of the corresponding parameters. Importantly, they involve a data transformation that translates the problem at hand into that of mean change point testing. To elaborate, the Hoeffding decomposition is an important tool to prove asymptotic results for $U$-statistics and shows equality between the original statistic and a sum of i.i.d. random variables (obtained from the Hajék projection) plus an asymptotically negligible remainder. Change point tests based on $U$-statistics were first proposed in the i.i.d. setting [50, 70, 81] and then generalised to weakly [57] and even long-range dependent data [29, [56]. Extensions based on $U$-quantiles are also available [55, 181]. Some first results for the data segmentation problem based on $U$ statistics already exist [59, 146], and Fearnhead and Rigaill [67] investigate the minimisation of penalised costs based on $M$-estimation via dynamic programming for change point analysis in the presence of outliers.

\section{Asymptotic embeddings}

Another extension is based on asymptotic results for partial sum processes such as functional central limit theorems or (strong) invariance principles [28, 82, 87, 117, 118, 121, 168]. Most importantly, such results enable the investigation into change point analysis of stochastic processes including multivariate Wiener processes with drift, renewal processes or integrated diffusion processes. A univariate version of such a model was introduced by Horváth and Steinebach [90] for change point testing against the AMOC alternative, and has been extended to multiple change point problems in Kühn [122] where the consistency is established for the estimator of the number of change points based on the SC described in Section 3.1.1. For the specific example of univariate renewal processes, Messer et al. [141] propose a multiscale MOSUM procedure with bandwidths linear in sample size (see Sections 3.2.2 and 3.2.4). Kirch and Klein [114] investigate a MOSUM procedure in the general multivariate framework adopting both linear and sub-linear bandwidths and derive localisation rates for the change point estimators that are shown to be minimax optimal when there are a finite number of change points; when there are an unbounded number of change points, this remains to be valid for Wiener processes with drift. Beyond such settings, minimax optimality in the localisation rate has not been established to the best of our knowledge (see also Section 2). 


\section{Nonparametric change point analysis}

Truly nonparametric change point tests do not have obvious connections to the canonical mean change point detection problem. These tests often involve appropriately weighted integrals over differences of the estimators of the nonparametric quantity of interest, such as those based on functionals of two-sample rank-based test statistics [191], empirical characteristic function [99, 100], and kernel estimators of nonparametric conditional mean functions [142] among others. In principle, local test methods for the canonical data segmentation problem discussed in Section 3.2, such as (W)BS or the MOSUM procedure, can be adapted to accommodate such tests, since they are based on localised applications of tests designed for the AMOC setting. Application of the global optimisation approaches to nonparametric settings is more involved, although some attempts exist [196].

\subsection{High-dimensional change point analysis}

Recent years have seen a surge of interest in the development of change point methodologies for high-dimensional data including functional and panel data and networks, with many papers addressing the problem of change point testing in the mean of panel data, see e.g. Sections 7 and 8 of the review paper by Horváth and Rice [89]. In this section, our aim is to show that the challenge due to high dimensionality of the data is orthogonal to that due to the presence of multiple change points in the following sense:

(i) As argued in Section 2, the goal of the canonical data segmentation problem is to achieve consistency in multiple change point detection even in difficult situations arising from the multiscale nature of the change points (see Definition 2.1 (a). In particular, the interplay between the spacing between change points, $\delta_{j}=\min \left(\theta_{j}-\theta_{j-1}, \theta_{j+1}-\theta_{j}\right)$, and the magnitude of changes, $\left|d_{j}\right|$, in their detectability is well-understood under (1). Accordingly, the methodological challenge lies in isolating each change point within an interval that is long enough for its detection and localisation.

(ii) In high-dimensional change point problems, the main question is how to boost the signal (on each change) across large dimensions. Adopting a minimax-viewpoint, this is closely related to the choice of the norm adopted for measuring the cross-sectional size of the change, which is highly relevant in high-dimensional settings since, unlike in univariate or even multivariate situations, standard norms are no longer equivalent.

Clearly, these two aspects, multiple change point detection and high-dimensional change point analysis, merit separate attention when developing a methodology that is most suitable for the problem at hand. There already exist papers addressing the problem of multiple change point detection in high-dimensional settings through combining the data segmentation algorithms for the canonical data segmentation problem with methods for cross-sectional aggregation of information on changes. We provide an incomplete review of the literature below. 
The application of the BS algorithm has been investigated in panel data segmentation problems based on first- [40] and second-order [42] properties, as well as under a factor model [24]. The WBS algorithm has been adopted for multiple change point detection in the mean [188] and the covariance [186] of high-dimensional panel data in combination with datadriven projections, and also adopted in regression settings [183]. Chu and Chen [48] and Liu and Chen 132 propose graph-based change point tests for non-Euclidean and possibly high-dimensional data, and the latter paper heuristically discusses their extensions to data segmentation problems in combination with BS and WBS. Chen et al. 39] adopt the MOSUM procedure for panel data segmentation in the mean, and a MOSUM-type screening strategy has also been adopted in Zhao et al. [195] for change point detection in dynamic networks. The $\ell_{0}$-penalisation method has been adopted in parametric settings such as regression [109, 126] and vector autoregressive [184 models and the $\ell_{1}$-penalisation method, together with an information criterion-based model selection, has been applied to high-dimensional time series segmentation under vector autoregressive models [22, 163].

We further elaborate on the issue discussed in (ii) above by focusing on the problem that has seen the most development, namely the problem of detecting changes in the mean of panel data under the following model:

$$
X_{i t}=f_{i t}+\varepsilon_{i t}=f_{i 0}+\sum_{j=1}^{q_{n}} d_{i j} \cdot \mathbb{I}_{t \geq \theta_{j}+1}+\varepsilon_{i t}, \quad i=1, \ldots, p, t=1, \ldots, n .
$$

It extends the canonical model (1) to the panel data setting with the vector $\mathbf{d}_{j}=\left(d_{i j}, \ldots, d_{p}\right)^{\top}$ denoting the change in the mean of $\mathbf{X}_{t}=\left(X_{1 t}, \ldots, X_{p t}\right)^{\top}$ at $t=\theta_{j}$, and $\varepsilon_{t}=\left(\varepsilon_{1 t}, \ldots, \varepsilon_{p t}\right)^{\top}$ is assumed to satisfy $\mathrm{E}\left(\varepsilon_{t}\right)=\mathbf{0}$ and $\operatorname{Cov}\left(\varepsilon_{t}\right)=\boldsymbol{\Sigma}$. Unlike in the multivariate setting, the dimensionality of the data $p=p_{n}$ depends on the sample size $n$ and is even allowed to increase faster than $n$.

Detectability of a change point depends on the magnitude of the corresponding change, and selecting a norm for measuring the magnitude of $\mathbf{d}_{j}$ effectively corresponds to modelling the (cross-sectional) type of a change for which the proposed change point methodology is particularly tailored. High-dimensional efficiency of change point tests, as proposed in Aston and Kirch [14], relates to the separation rate between detectable and undetectable changes. The norms associated with the high-dimensional efficiency of different methods proposed in the existing literature indeed vary: Methods designed for detecting sparse changes (where $\left.\left\|\mathbf{d}_{j}\right\|_{0}:=\sum_{i=1}^{p} \mathbb{I}_{\left|d_{i j}\right|>0} \ll p\right)$ such as those proposed in Jirak [108, Cho and Fryzlewicz [42], Wang and Samworth [188], Chen et al. [39] and the scan statistic of Enikeeva and Harchaoui [64], are associated with the $\ell_{\infty}$-norm (sometimes implicitly so via $\left\|\mathbf{d}_{j}\right\| / \sqrt{\left\|\mathbf{d}_{j}\right\|_{0}}$ ), while the methods from Horváth and Hušková [88, Wang et al. [187] and the linear statistic of Enikeeva and Harchaoui [64] are associated with the $\ell_{2}$-norm, see Table 2 for a summary which extends Table 1 of Cho [40]. 
Table 2: Comparison of high-dimensional change point detection methods for the problem (11) on their high-dimensional efficiency [14] when $q_{n}=1, \min \left(\theta_{1}, n-\theta_{1}\right) \geq c n$ for some $c \in(0,1 / 2$ ] and $\boldsymbol{\Sigma}$ is diagonal with all the variances of the same order, i.e. $0<\sigma_{\min }^{2} \leq \operatorname{Var}\left(\varepsilon_{i t}\right) \leq \sigma_{\max }^{2}<$ $\infty$ for all $i=1, \ldots, p$. The efficiency, denoted by $\mathcal{E}\left(\mathbf{d}_{1}\right)$, indicates that the corresponding procedure has asymptotic power one if $\sqrt{n} \mathcal{E}\left(\mathbf{d}_{1}\right) \rightarrow \infty$. By $L_{n, p}$, we denote a term logarithmic in $n$ and/or $p$ which varies from one instance to another.

\begin{tabular}{l|c||l|c}
\hline \hline [14]: oracle & $\left\|\mathbf{d}_{1}\right\|$ & [14]: random & $p^{-1 / 2}\left\|\mathbf{d}_{1}\right\|$ \\
\hline [88, 187] & $p^{-1 / 4}\left\|\mathbf{d}_{1}\right\|$ & [42, 108, 39] & $L_{n, p}^{-1}\left\|\mathbf{d}_{1}\right\|_{\infty}$ \\
\hline 64]: linear & $\left(p^{1 / 4} L_{n, p}\right)^{-1}\left\|\mathbf{d}_{1}\right\|$ & [64]: scan, [188] & $\left(\left\|\mathbf{d}_{1}\right\|_{0} L_{n, p}\right)^{-1 / 2}\left\|\mathbf{d}_{1}\right\|$ \\
\hline
\end{tabular}

Minimax optimality is also linked to the choice of norm, in the sense that minimax optimality of a given procedure with respect to a norm indicates that the procedure is optimal in the worst possible case defined by that particular norm. On the other hand, this does not provide much information as to how the procedure performs in the worst case scenarios defined with different norms. For example, the test proposed by Horváth and Hušková [88] is minimax optimal with respect to the $\ell_{2}$-norm, when the panel is spatially independent and all variances are of the same order (in the sense described in Table 2), see the discussion below Theorem 2.1 of [14]. The test statistic proposed therein increases the signal-to-noise ratio (while controlling for the size) by summing up squared change point statistics; as more cross-sectional components are considered, the signal increases according to the squared $\ell_{2}$-norm, i.e. linear in the number of components with a change, whereas the noise only increases as $\sqrt{p}$ due to the central limit theorem applicable under the spatial independence assumption. However, it does not adapt to the sparsity of change or match the minimax optimal rate with respect to the $\ell_{\infty}$-norm; we refer to Liu et al. 131] where the authors show the presence of a phase transition boundary for the sparsity in the minimax rate of the high-dimensional change testing problem under (11) (i.e. $\left.q_{n} \leq 1\right)$.

The difficulty in high-dimensional data analysis is that spurious low-dimensional signals appear simply by chance, another flavour of the curse of dimensionality which needs to be accounted for in any data science problem. Under (11) with a single change point, the direction of a change, $\mathbf{d}_{1}$, is a one-dimensional object such that the projection of $\mathbf{X}_{t}$ with respect to the 'oracle' projection vector $\mathbf{p}_{o}=\boldsymbol{\Sigma}^{-1} \mathbf{d}_{1}$ yields the optimal signal-to-noise ratio, see Proposition 3.3 of [14]. In practice, however, neither $\mathbf{d}_{1}$ nor $\boldsymbol{\Sigma}$ are known, and maximising over all possible projections $\mathbf{p} \in \mathbb{R}^{p}$ will lead to too large a noise level due to the above observation. Random projections (independent of the data) have been considered as an alternative, see Steland [169] addressing the problem of change point testing in the covariance structure. However, this is at the cost of the loss of detection power as illustrated by the highdimensional efficiency of such random projections, see Table 2 which shows that a random projection is a magnitude of $p^{-1 / 2}$ worse than oracle efficiency when $\boldsymbol{\Sigma}$ is a diagonal matrix, and also Theorem 3.5 of 14 .

Data-driven projections such as those proposed in [39, 42, 64, 188, can keep the increased noise 
level at bay, by effectively working under the sparsity assumption on $\mathbf{d}_{j}$ and consequently considering only a subset of all possible projections. This comes at the cost of decreased high-dimensional efficiency when the sparsity assumption is not met, compared to the oracle projection with respect to $\mathbf{p}_{\mathrm{o}}$ or the $\ell_{2}$-norm minimax optimal procedures such as [88, 187]. Compared to the panel data setting, change point analysis in functional data is benefited by the additional structure implied by the assumption of observing a random function, which helps lifting part of the curse of dimensionality. Some approaches [13, 27] to the problem of detecting changes in the mean function are based on dimension reduction e.g. via functional principal component analysis. Another line of research proposes 'fully functional' procedures [18, 166] in the sense that they do not employ any dimension reduction. Stoehr et al. 170] propose a compromise between the two approaches in the context of change point detection in the covariance structure.

\section{Conclusions}

In this paper, we first review and compare different approaches to the canonical segmentation problem where the aim is to estimate the number and the locations of multiple change points in the mean of univariate data. In Section 3.3, we provide an overview of representative methodologies comparing them according to the theoretical framework given in Section 2, and briefly comment on their numerical performance based on comparative simulation studies on a range of test signals.

In the second part of the paper, we give a survey on topics such as change point analysis beyond the detection of mean changes under Gaussianity (Section 4.1), and high-dimensional change point analysis (Section 4.2). In Section 4.1, we argue that change point tests developed for more complex problems are closely related to the mean change point problem and make the connection transparent. As discussed in Section 2, some of the methods for the canonical data segmentation problem are based on localised applications of the tests for the AMOC alternative, and thus can be applied to more complex change point problems in combination with corresponding tests. Clearly, theoretical underpinnings of the combined methodology depend on those of the individual components. In Section 4.2 , we show that in high-dimensional change point analysis, challenges arising from the high dimensionality are orthogonal to those arising from the presence of multiple change points and, based on indepth understanding of the component problems, methods addressing individual aspects can be combined to best address the given problems. Finally, combining the elements from the three fields opens up a new avenue for future research, namely the development of statistical methodologies for complex, high-dimensional data segmentation problems. 


\section{References}

[1] Niall M Adams and Nicholas Heard. Dynamic Networks and Cyber-security, volume 1. World Scientific, 2016.

[2] Samaneh Aminikhanghahi and Diane J Cook. A survey of methods for time series change point detection. Knowledge and Information Systems, 51:339-367, 2017.

[3] Andreas Anastasiou and Piotr Fryzlewicz. IDetect: Detecting multiple generalized change-points by isolating single ones, 2018. URL https://CRAN . R-project .org/package=IDetect. R package version 1.0.

[4] Andreas Anastasiou and Piotr Fryzlewicz. Detecting multiple generalized change-points by isolating single ones. Metrika, pages 1-34, 2021.

[5] Andreas Anastasiou, Yining Chen, Haeran Cho, and Piotr Fryzlewicz. breakfast: Methods for Fast Multiple Change-Point Detection and Estimation, 2021. URL https://CRAN.R-project. org/package=breakfast. $R$ package version 2.2.

[6] Jaromír Antoch and Marie Hušková. Procedures for the detection of multiple changes in series of independent observations. In Asymptotic Statistics, pages 3-20. Springer, 1994.

[7] Jaromir Antoch and Marie Hušková. Estimators of changes. In Asymptotics, Nonparametrics, and Time Series, pages 557-561. CRC Press, 1999.

[8] Jaromır Antoch and Marie Hušková. Permutation tests in change point analysis. Statistics \& Probability Letters, 53(1):37-46, 2001.

[9] Jaromir Antoch, Marie Hušková, and Noël Veraverbeke. Change-point problem and bootstrap. Journal of Nonparametric Statistics, 5(2):123-144, 1995.

[10] Ery Arias-Castro, Emmanuel J Candes, and Arnaud Durand. Detection of an anomalous cluster in a network. The Annals of Statistics, 39:278-304, 2011.

[11] Sylvain Arlot and Alain Celisse. Segmentation of the mean of heteroscedastic data via crossvalidation. Statistics and Computing, 21(4):613-632, 2011.

[12] Sylvain Arlot, Alain Celisse, and Zaid Harchaoui. A kernel multiple change-point algorithm via model selection. Journal of Machine Learning Research, 20(162), 2019.

[13] John AD Aston and Claudia Kirch. Detecting and estimating changes in dependent functional data. Journal of Multivariate Analysis, 109:204-220, 2012.

[14] John AD Aston and Claudia Kirch. High dimensional efficiency with applications to change point tests. Electronic Journal of Statistics, 12:1901-1947, 2018.

[15] Alexander Aue and Lajos Horváth. Structural breaks in time series. Journal of Time Series Analysis, 34:1-16, 2013.

[16] Alexander Aue, Siegfried Hörmann, Lajos Horváth, and Matthew Reimherr. Break detection in the covariance structure of multivariate time series models. The Annals of Statistics, 37: 4046-4087, 2009.

[17] Alexander Aue, Rex CY Cheung, Thomas CM Lee, and Ming Zhong. Segmented model selection in quantile regression using the minimum description length principle. Journal of the American Statistical Association, 109:1241-1256, 2014.

[18] Alexander Aue, Gregory Rice, and Ozan Sönmez. Detecting and dating structural breaks in functional data without dimension reduction. Journal of the Royal Statistical Society, Series B, 80:509-529, 2018.

[19] Ivan E Auger and Charles E Lawrence. Algorithms for the optimal identification of segment neighborhoods. Bulletin of Mathematical Biology, 51:39-54, 1989.

[20] Ieva Axt and Roland Fried. On variance estimation under shifts in the mean. ASta Advanced in Statistical Analysis, 104:417-457, 2020.

[21] Jushan Bai and Pierre Perron. Estimating and testing linear models with multiple structural changes. Econometrica, pages 47-78, 1998.

[22] Peiliang Bai, Abolfazl Safikhani, and George Michailidis. Multiple change points detection in low rank and sparse high dimensional vector autoregressive models. IEEE Transactions on Signal Processing, 68:3074-3089, 2020.

[23] Rafal Baranowski, Yining Chen, and Piotr Fryzlewicz. Narrowest-over-threshold detection of 
multiple change-points and change-point-like features. Journal of the Royal Statistical Society, Series B, 81:649-672, 2019.

[24] Matteo Barigozzi, Haeran Cho, and Piotr Fryzlewicz. Simultaneous multiple change-point and factor analysis for high-dimensional time series. Journal of Econometrics, 206:187-225, 2018.

[25] Daniel Barry and John A Hartigan. A Bayesian analysis for change point problems. Journal of the American Statistical Association, 88:309-319, 1993.

[26] Simon Behrendt and Karsten Schweikert. A note on adaptive group Lasso for structural break time series. Econometrics and Statistics, 17:156-172, 2021.

[27] István Berkes, Robertas Gabrys, Lajos Horváth, and Piotr Kokoszka. Detecting changes in the mean of functional observations. Journal of the Royal Statistical Society, Series B, 71:927-946, 2009.

[28] István Berkes, Weidong Liu, and Wei Biao Wu. Komlós-Major-Tusnády approximation under dependence. The Annals of Probability, 42:794-817, 2014.

[29] Annika Betken. Testing for change-points in long-range dependent time series by means of a self-normalized wilcoxon test. Journal of Time Series Analysis, 37(6):785-809, 2016.

[30] Leif Boysen, Angela Kempe, Volkmar Liebscher, Axel Munk, and Olaf Wittich. Consistencies and rates of convergence of jump-penalized least squares estimators. The Annals of Statistics, $37: 157-183,2009$.

[31] B. E. Brodsky and B. S. Darkhovsky. Nonparametric Statistical Diagnosis: Problems and Methods, volume 509. Springer Science \& Business Media, 2000.

[32] Axel Bücher, Jean-David Fermanian, and Ivan Kojadinovic. Combining cumulative sum changepoint detection tests for assessing the stationarity of univariate time series. Journal of Time Series Analysis, 40(1):124-150, 2019.

[33] Alain Celisse, Guillemette Marot, Morgane Pierre-Jean, and GJ Rigaill. New efficient algorithms for multiple change-point detection with reproducing kernels. Computational Statistics \& Data Analysis, 128:200-220, 2018.

[34] Souhil Chakar, E Lebarbier, Céline Lévy-Leduc, and Stéphane Robin. A robust approach for estimating change-points in the mean of an AR(1) process. Bernoulli, 23(2):1408-1447, 2017.

[35] Hock Peng Chan and Hao Chen. Multi-sequence segmentation via score and higher-criticism tests. arXiv preprint, arXiv:1706.07586, 2017.

[36] Ngai Hang Chan, Chun Yip Yau, and Rong-Mao Zhang. Group Lasso for structural break time series. Journal of the American Statistical Association, 109:590-599, 2014.

[37] Jiahua Chen, AK Gupta, and Jianmin Pan. Information criterion and change point problem for regular models. Sankhyā: The Indian Journal of Statistics, 68:252-282, 2006.

[38] Jie Chen and Arjun K Gupta. Parametric statistical change point analysis: with applications to genetics, medicine, and finance. Springer Science \& Business Media, 2nd edition, 2011.

[39] Likai Chen, Weining Wang, and Weibiao Wu. Inference of break-points in high-dimensional time series. Journal of the American Statistical Association (to appear), 2021.

[40] Haeran Cho. Change-point detection in panel data via double CUSUM statistic. Electronic Journal of Statistics, 10:2000-2038, 2016.

[41] Haeran Cho and Piotr Fryzlewicz. Multiscale and multilevel technique for consistent segmentation of nonstationary time series. Statistica Sinica, 22:207-229, 2012.

[42] Haeran Cho and Piotr Fryzlewicz. Multiple change-point detection for high-dimensional time series via sparsified binary segmentation. Journal of the Royal Statistical Society, Series B, 77: 475-507, 2015.

[43] Haeran Cho and Piotr Fryzlewicz. Corrections on 'multiple change-point detection for highdimensional time series via sparsified binary segmentation'. https://people.maths.bris.ac . uk/ mahrc/papers/sbs_correction.pdf, 2015.

[44] Haeran Cho and Piotr Fryzlewicz. Multiple change point detection under serial dependence: Wild energy maximisation and gappy Schwarz criterion. arXiv preprint arXiv:2011.13884, 2020.

[45] Haeran Cho and Claudia Kirch. Two-stage data segmentation permitting multiscale change points, heavy tails and dependence. arXiv preprint arXiv:1910.12486, 2020.

[46] Haeran Cho and Claudia Kirch. Bootstrap confidence intervals for multiple change points based 
on moving sum procedures. arXiv preprint arXiv:2106.12844, 2021.

[47] Chia-Shang J Chu, Kurt Hornik, and Chung-Ming Kaun. MOSUM tests for parameter constancy. Biometrika, 82:603-617, 1995.

[48] Lynna Chu and Hao Chen. Asymptotic distribution-free change-point detection for multivariate and non-Euclidean data. The Annals of Statistics, 47:382-414, 2019.

[49] Alice Cleynen, Guillem Rigaill, and Michel Koskas. Segmentor3IsBack: A fast segmentation algorithm, 2016. URL https://CRAN.R-project.org/package=Segmentor3IsBack. R package version 2.0 .

[50] Miklós Csörgő and Lajos Horváth. Invariance principles for changepoint problems. In Multivariate statistics and probability, pages 151-168. Elsevier, 1989.

[51] Miklós Csörgö and Lajos Horváth. Limit Theorems in Change-point Analysis, volume 18. John Wiley \& Sons Inc, 1997.

[52] Richard A Davis and Chun Yip Yau. Consistency of minimum description length model selection for piecewise stationary time series models. Electronic Journal of Statistics, 7:381-411, 2013.

[53] Richard A Davis, Thomas C M Lee, and Gabriel A Rodriguez-Yam. Structural break estimation for nonstationary time series models. Journal of the American Statistical Association, 101: 223-239, 2006.

[54] Richard A Davis, Thomas CM Lee, and Gabriel A Rodriguez-Yam. Break detection for a class of nonlinear time series models. Journal of Time Series Analysis, 29:834-867, 2008.

[55] H Dehling, R Fried, and M Wendler. A robust method for shift detection in time series. Biometrika, 107(3):647-660, 2020.

[56] Herold Dehling, Aeneas Rooch, and Murad S Taqqu. Non-parametric change-point tests for long-range dependent data. Scandinavian Journal of Statistics, 40(1):153-173, 2013.

[57] Herold Dehling, Roland Fried, Isabel Garcia, and Martin Wendler. Change-point detection under dependence based on two-sample U-statistics. In Asymptotic Laws and Methods in Stochastics, pages 195-220. Springer, 2015.

[58] Holger Dette, Theresa Schüler, and Mathias Vetter. Multiscale change point detection for dependent data. Scandinavian Journal of Statistics, 47:1243-1274, 2020.

[59] Maik Döring. Multiple change-point estimation with U-statistics. Journal of Statistical Planning and Inference, 140(7):2003-2017, 2010.

[60] Chao Du, Chu-Lan Michael Kao, and SC Kou. Stepwise signal extraction via marginal likelihood. Journal of the American Statistical Association, 111:314-330, 2016.

[61] Idris A Eckley, Paul Fearnhead, and Rebecca Killick. Analysis of changepoint models. In David Barber, A. Taylan Cemgil, and Silvia Chiappa, editors, Bayesian Time Series Models, pages 205-224. Cambridge University Press, 2011.

[62] Bradley Efron, Trevor Hastie, Iain Johnstone, and Robert Tibshirani. Least angle regression. The Annals of Statistics, 32:407-499, 2004.

[63] Birte Eichinger and Claudia Kirch. A MOSUM procedure for the estimation of multiple random change points. Bernoulli, 24:526-564, 2018.

[64] Farida Enikeeva and Zaid Harchaoui. High-dimensional change-point detection under sparse alternatives. The Annals of Statistics, 47:2051-2079, 2019.

[65] Xiao Fang, Jian Li, and David Siegmund. Segmentation and estimation of change-point models: false positive control and confidence regions. The Annals of Statistics, 48:1615-1647, 2020.

[66] Paul Fearnhead. Exact and efficient Bayesian inference for multiple changepoint problems. Statistics and Computing, 16:203-213, 2006.

[67] Paul Fearnhead and Guillem Rigaill. Changepoint detection in the presence of outliers. Journal of the American Statistical Association, 114:169-183, 2019.

[68] Paul Fearnhead and Guillem Rigaill. Relating and comparing methods for detecting changes in mean. Stat, page e291, 2020.

[69] Paul Fearnhead, Robert Maidstone, and Adam Letchford. Detecting changes in slope with an $l_{0}$ penalty. Journal of Computational and Graphical Statistics, 28:265-275, 2019.

[70] Dietmar Ferger. On the power of nonparametric changepoint-tests. Metrika, 41(1):277-292, 1994. 
[71] Alexander Tristan Maximilian Fisch, Idris Arthur Eckley, and Paul Fearnhead. A linear time method for the detection of point and collective anomalies. arXiv preprint arXiv:1806.01947, 2018.

[72] Klaus Frick, Axel Munk, and Hannes Sieling. Multiscale change point inference. Journal of the Royal Statistical Society, Series B, 76:495-580, 2014.

[73] M. Fromont, M. Lerasle, and N. Verzelen. Optimal change point detection and localization. arXiv preprint arXiv:2010.11470, 2020.

[74] Piotr Fryzlewicz. Wild binary segmentation for multiple change-point detection. The Annals of Statistics, 42:2243-2281, 2014.

[75] Piotr Fryzlewicz. Tail-greedy bottom-up data decompositions and fast multiple change-point detection. The Annals of Statistics, pages 3390-3421, 2018.

[76] Piotr Fryzlewicz. Detecting possibly frequent change-points: Wild Binary Segmentation 2 and steepest-drop model selection. Journal of the Korean Statistical Society, 49:1-44, 2020.

[77] Piotr Fryzlewicz. Narrowest significance pursuit: inference for multiple change-points in linear models. arXiv preprint arXiv:2009.05431, 2020.

[78] Piotr Fryzlewicz and Suhasini Subba Rao. Multiple-change-point detection for auto-regressive conditional heteroscedastic processes. Journal of the Royal Statistical Society, Series B, 76: 903-924, 2014.

[79] Cheng-Der Fuh. Asymptotic operating characteristics of an optimal change point detection in hidden Markov models. The Annals of Statistics, 32:2305-2339, 2004.

[80] Damien Garreau and Sylvain Arlot. Consistent change-point detection with kernels. Electronic Journal of Statistics, 12:4440-4486, 2018.

[81] Edit Gombay. U-statistics for change under alternatives. Journal of Multivariate Analysis, 78 (1):139-158, 2001.

[82] Tomasz Górecki, Lajos Horváth, and Piotr Kokoszka. Change point detection in heteroscedastic time series. Econometrics and Statistics, 7:63-88, 2018.

[83] Peter Grunwald. A tutorial introduction to the minimum description length principle. arXiv preprint arXiv:math/0406077, 2004.

[84] Zard Harchaoui and Céline Lévy-Leduc. Multiple change-point estimation with a total variation penalty. Journal of the American Statistical Association, 105:1480-1493, 2010.

[85] Kaylea Haynes, Idris A Eckley, and Paul Fearnhead. Computationally efficient changepoint detection for a range of penalties. Journal of Computational and Graphical Statistics, 26:134$143,2017$.

[86] Kaylea Haynes, Paul Fearnhead, and Idris A Eckley. A computationally efficient nonparametric approach for changepoint detection. Statistics and Computing, 27:1293-1305, 2017.

[87] A. J. Heunis. Strong invariance principle for singular diffusions. Stochastic Processes and their Applications, 104:57-80, 2003.

[88] L. Horváth and M. Hušková. Change-point detection in panel data. Journal of Time Series Analysis, 33:631-648, 2012.

[89] Lajos Horváth and Gregory Rice. Extensions of some classical methods in change point analysis. TEST, 23:1-37, 2014.

[90] Lajos Horváth and Josef Steinebach. Testing for changes in the mean or variance of a stochastic process under weak invariance. Journal of Statistical Planning and Inference, 91:365-376, 2000.

[91] Marie Hušková. Asymptotics for robust MOSUM. Commentationes Mathematicae Universitatis Carolinae, 31(2):345-356, 1990.

[92] Marie Hušková. Some asymptotic results for robust procedures for testing the constancy of regression models over time. Kybernetika, 26(5):392-403, 1990.

[93] Marie Hušková. Tests and estimators for the change point problem based on M-statistics. Statistics $\mathcal{G}$ Risk Modeling, 14:115-136, 1996.

[94] Marie Hušková. Permutation principle and bootstrap in change point analysis. In L. Horváth and B. Szyszkowicz, editors, Asymptotic Methods in Stochastics. Festschrift for Miklós Csörgö, volume 44, pages 273-292. American Mathematical Society, 2004.

[95] Marie Hušková. Robust change point analysis. In Robustness and Complex Data Structures, 
pages 171-190. Springer, 2013.

[96] Marie Hušková and Claudia Kirch. Bootstrapping confidence intervals for the change-point of time series. Journal of Time Series Analysis, 29:947-972, 2008.

[97] Marie Hušková and Claudia Kirch. A note on studentized confidence intervals for the changepoint. Computational Statistics, 25:269-289, 2010.

[98] Marie Hušková and Miriam Marušiaková. M-procedures for detection of changes for dependent observations. Communications in Statistics - Simulation and Computation, 41(7):1032-1050, 2012.

[99] Marie Hušková and Simos G Meintanis. Change point analysis based on empirical characteristic functions. Metrika, 63:145-168, 2006.

[100] Marie Hušková and Simos G Meintanis. Change-point analysis based on empirical characteristic functions of ranks. Sequential Analysis, 25(4):421-436, 2006.

[101] Marie Hušková and Jan Picek. M-tests for detection of structural changes in regression. In Statistical data analysis based on the L1-norm and related methods, pages 213-227. Springer, 2002.

[102] Marie Hušková and Aleš Slabỳ. Permutation tests for multiple changes. Kybernetika, 37:605-622, 2001.

[103] Marie Hušková, Zuzana Prášková, and Josef Steinebach. On the detection of changes in autoregressive time series I. Asymptotics. Journal of Statistical Planning and Inference, 137:1243-1259, 2007.

[104] Sangwon Hyun, Kevin Z Lin, Max G'Sell, and Ryan J Tibshirani. Post-selection inference for changepoint detection algorithms with application to copy number variation data. Biometrics, 2021.

[105] Brad Jackson, Jeffrey D Scargle, David Barnes, Sundararajan Arabhi, Alina Alt, Peter Gioumousis, Elyus Gwin, Paungkaew Sangtrakulcharoen, Linda Tan, and Tun Tao Tsai. An algorithm for optimal partitioning of data on an interval. IEEE Signal Processing Letters, 12:105-108, 2005.

[106] Venkata Jandhyala, Stergios Fotopoulos, Ian MacNeill, and Pengyu Liu. Inference for single and multiple change-points in time series. Journal of Time Series Analysis, 34(4):423-446, 2013.

[107] Sean Jewell, Paul Fearnhead, and Daniela Witten. Testing for a change in mean after changepoint detection. arXiv preprint arXiv:1910.04291, 2019.

[108] Moritz Jirak. Uniform change point tests in high dimension. The Annals of Statistics, 43: 2451-2483, 2015.

[109] Abhishek Kaul, Venkata K Jandhyala, and Stergios B Fotopoulos. Detection and estimation of parameters in high dimensional multiple change point regression models via $\ell_{1} / \ell_{0}$ regularization and discrete optimization. arXiv preprint arXiv:1906.04396, 2019.

[110] Rebecca Killick, Paul Fearnhead, and Idris A Eckley. Optimal detection of changepoints with a linear computational cost. Journal of the American Statistical Association, 107:1590-1598, 2012.

[111] Rebecca Killick, Kaylea Haynes, and Idris A. Eckley. changepoint: An R package for changepoint analysis, 2016. URL https://CRAN.R-project.org/package=changepoint. R package version 2.2.2.

[112] Claudia Kirch and J Tadjuidje Kamgaing. Detection of change points in discrete valued time series. In Richard A. Davis, Scott H. Holan, Robert Lund, and Nalini Ravishanker, editors, Handbook of Discrete Valued Time Series. Chapman and Hall/CRC, New York, 2014.

[113] Claudia Kirch and Joseph Tadjuidje Kamgaing. Testing for parameter stability in nonlinear autoregressive models. Journal of Time Series Analysis, 33:365-385, 2012.

[114] Claudia Kirch and Philipp Klein. Moving sum data segmentation for stochastics processes based on invariance. arXiv preprint arXiv:2101.04651, 2021.

[115] Claudia Kirch and Kerstin Reckrühm. Data segmentation for time series based on a general moving sum approach. In preparation, 2021.

[116] Claudia Kirch, Birte Muhsal, and Hernando Ombao. Detection of changes in multivariate time series with application to EEG data. Journal of the American Statistical Association, 110: 1197-1216, 2015 . 
[117] János Komlós, Péter Major, and Gábor Tusnády. An approximation of partial sums of independent RV's, and the sample DF. I. Zeitschrift für Wahrscheinlichkeitstheorie und verwandte Gebiete, 32:111-131, 1975.

[118] János Komlós, Péter Major, and Gábor Tusnády. An approximation of partial sums of independent RV's, and the sample DF. II. Zeitschrift für Wahrscheinlichkeitstheorie und verwandte Gebiete, 34:33-58, 1976.

[119] A. Korostelev. On minimax estimation of a discontinuous signal. Theory of Probability and its Applications, 32:727-730, 1987.

[120] Solt Kovács, Housen Li, Peter Bühlmann, and Axel Munk. Seeded binary segmentation: A general methodology for fast and optimal change point detection. arXiv preprint arXiv:2002.06633, 2020.

[121] James Kuelbs and Walter Philipp. Almost sure invariance principles for partial sums of mixing $B$-valued random variables. The Annals of Probability, 8:1003-1036, 1980.

[122] Christoph Kühn. An estimator of the number of change points based on a weak invariance principle. Statistics \& Probability Letters, 51:189-196, 2001.

[123] Marc Lavielle and Eric Moulines. Least-squares estimation of an unknown number of shifts in a time series. Journal of Time Series Analysis, 21:33-59, 2000.

[124] Émilie Lebarbier. Detecting multiple change-points in the mean of Gaussian process by model selection. Signal Processing, 85(4):717-736, 2005.

[125] Chung-Bow Lee. Estimating the number of change points in a sequence of independent normal random variables. Statistics \& Probability Letters, 25:241-248, 1995.

[126] Florencia Leonardi and Peter Bühlmann. Computationally efficient change point detection for high-dimensional regression. arXiv preprint arXiv:1601.03704, 2016.

[127] Housen Li and Hannes Sieling. FDRSeg: FDR-control in multiscale change-point segmentation, 2017. URL https://CRAN.R-project.org/package=FDRSeg. R package version 1.0-3.

[128] Housen Li, Axel Munk, and Hannes Sieling. FDR-control in multiscale change-point segmentation. Electronic Journal of Statistics, 10:918-959, 2016.

[129] Housen Li, Qinghai Guo, and Axel Munk. Multiscale change-point segmentation: Beyond step functions. Electronic Journal of Statistics, 13:3254-3296, 2019.

[130] Kevin Lin, James L Sharpnack, Alessandro Rinaldo, and Ryan J Tibshirani. A sharp error analysis for the fused Lasso, with application to approximate changepoint screening. In Advances in Neural Information Processing Systems, pages 6884-6893, 2017.

[131] Haoyang Liu, Chao Gao, and Richard J Samworth. Minimax rates in sparse, high-dimensional change point detection. The Annals of Statistics, 49(2):1081-1112, 2021.

[132] Yi-Wei Liu and Hao Chen. A fast and efficient change-point detection framework for modern data. arXiv preprint arXiv:2006.13450, 2020.

[133] Zhiyuan Lu, Moulinath Banerjee, and George Michailidis. Intelligent sampling for multiple change-points in exceedingly long time series with rate guarantees. arXiv preprint arXiv:1710.07420, 2020.

[134] Hyeyoung Maeng and Piotr Fryzlewicz. Detecting linear trend changes and point anomalies in data sequences. arXiv preprint arXiv:1906.01939, 2019.

[135] Robert Maidstone, Toby Hocking, Guillem Rigaill, and Paul Fearnhead. On optimal multiple changepoint algorithms for large data. Statistics and Computing, 27:519-533, 2017.

[136] Miriam Marušiaková. Tests for multiple changes in linear regression models. PhD thesis, Charles University in Prague, 2009.

[137] David S. Matteson and Nicholas A. James. A nonparametric approach for multiple change point analysis of multivariate data. Journal of the American Statistical Association, 109:334-345, 2014.

[138] Alexander Meier, Haeran Cho, and Claudia Kirch. mosum: Moving sum based procedures for changes in the mean, 2021. URL https://CRAN.R-project.org/package=mosum. R package version 1.2.6.

[139] Alexander Meier, Claudia Kirch, and Haeran Cho. mosum: A package for moving sums in change-point analysis. Journal of Statistical Software, 97(1):1-42, 2021. 
[140] Michael Messer, Marietta Kirchner, Julia Schiemann, Jochen Roeper, Ralph Neininger, and Gaby Schneider. A multiple filter test for the detection of rate changes in renewal processes with varying variance. The Annals of Applied Statistics, 8:2027-2067, 2014.

[141] Michael Messer, Stefan Albert, and Gaby Schneider. The multiple filter test for change point detection in time series. Metrika, 81:589-607, 2018.

[142] Maria Mohr and Natalie Neumeyer. Consistent nonparametric change point detection combining CUSUM and marked empirical processes. Electronic Journal of Statistics, 14:2238-2271, 2020.

[143] Yoshiyuki Ninomiya. Information criterion for Gaussian change-point model. Statistics E Probability Letters, 72:237-247, 2005.

[144] Yue S Niu and Heping Zhang. The screening and ranking algorithm to detect DNA copy number variations. The Annals of Applied Statistics, 6:1306-1326, 2012.

[145] Adam B Olshen, ES Venkatraman, Robert Lucito, and Michael Wigler. Circular binary segmentation for the analysis of array-based DNA copy number data. Biostatistics, 5:557-572, 2004.

[146] M Orasch. Using U-statistcs based processes to detect multiple change-points. In L. Horváth and B. Szyszkowicz, editors, Asymptotic Methods in Stochastics. Festschrift for Miklós Csörgö, volume 44, pages 315-334. American Mathematical Society, 2004.

[147] Oscar Hernan Madrid Padilla, Yi Yu, Daren Wang, and Alessandro Rinaldo. Optimal nonparametric change point detection and localization. arXiv preprint arXiv:1905.10019, 2019.

[148] Ewan S Page. Continuous inspection schemes. Biometrika, 41:100-115, 1954.

[149] Jianmin Pan and Jiahua Chen. Application of modified information criterion to multiple change point problems. Journal of Multivariate Analysis, 97:2221-2241, 2006.

[150] Florian Pein, Hannes Sieling, and Axel Munk. Heterogeneous change point inference. Journal of the Royal Statistical Society, Series B, 79:1207-1227, 2017.

[151] Florian Pein, Thomas Hotz, Hannes Sieling, and Timo Aspelmeier. stepR: Multiscale changepoint inference, 2019. URL https://CRAN.R-project.org/package=stepR. R package version 2.0-3.

[152] Pierre Perron. Dealing with structural breaks. Palgrave Handbook of Econometrics, 1:278-352, 2006.

[153] Zuzana Prášková and Ondřej Chochola. M-procedures for detection of a change under weak dependence. Journal of Statistical Planning and Inference, 149:60-76, 2014.

[154] Philip Preuss, Ruprecht Puchstein, and Holger Dette. Detection of multiple structural breaks in multivariate time series. Journal of the American Statistical Association, 110:654-668, 2015.

[155] Kerstin Reckrühm. Estimating multiple structural breaks in time series - a generalized MOSUM approach based on estimating functions. PhD thesis, Otto-von-Guericke University, Magdeburg, Germany, 2019.

[156] Jaxk Reeves, Jien Chen, Xiaolan L Wang, Robert Lund, and Qi Qi Lu. A review and comparison of changepoint detection techniques for climate data. Journal of Applied Meteorology and Climatology, 46:900-915, 2007.

[157] Guillem Rigaill. A pruned dynamic programming algorithm to recover the best segmentations with 1 to K_max change-points. Journal de la Société Frankcaise de Statistique, 156:180-205, 2015.

[158] Guillem Rigaill and Toby Dylan Hocking. fpop: Segmentation using Optimal Partitioning and Function Pruning, 2019. URL https://R-Forge.R-project.org/projects/opfp/. R package version 2019.01.22/r56.

[159] Guillem Rigaill, Émilie Lebarbier, and Stephane Robin. Exact posterior distributions and model selection criteria for multiple change-point detection problems. Statistics and Computing, 22: 917-929, 2012.

[160] Alessandro Rinaldo. Properties and refinements of the fused Lasso. The Annals of Statistics, $37: 2922-2952,2009$

[161] Jorma Rissanen. Modeling by shortest data description. Automatica, 14:465-471, 1978.

[162] Gaetano Romano, Guillem Rigaill, Vincent Runge, and Paul Fearnhead. Detecting abrupt changes in the presence of local fluctuations and autocorrelated noise. Journal of the American 
Statistical Association (to appear), 2021.

[163] Abolfazl Safikhani and Ali Shojaie. Joint structural break detection and parameter estimation in high-dimensional nonstationary VAR models. Journal of the American Statistical Association, pages 1-14, 2020.

[164] Gideon Schwarz. Estimating the dimension of a model. The Annals of Statistics, 6:461-464, 1978.

[165] Andrew Jhon Scott and M Knott. A cluster analysis method for grouping means in the analysis of variance. Biometrics, 6:507-512, 1974.

[166] Olimjon Sharipov, Johannes Tewes, and Martin Wendler. Sequential block bootstrap in a Hilbert space with application to change point analysis. Canadian Journal of Statistics, 44:300-322, 2016.

[167] Seung Jun Shin, Yichao Wu, and Ning Hao. A backward procedure for change-point detection with applications to copy number variation detection. Canadian Journal of Statistics, 48:366$385,2020$.

[168] J. Steinebach and V. R. Eastwood. Extreme value asymptotics for multivariate renewal processes. Journal of Multivariate Analysis, 56:284-302, 1996.

[169] Ansgar Steland. Testing and estimating change-points in the covariance matrix of a highdimensional time series. Journal of Multivariate Analysis, 177:104582, 2020.

[170] Christina Stoehr, John AD Aston, and Claudia Kirch. Detecting changes in the covariance structure of functional time series with application to fmri data. Econometrics and Statistics, 18:44-62, 2021.

[171] Alexander Tartakovsky. Sequential Change Detection and Hypothesis Testing: General Non-iid Stochastic Models and Asymptotically Optimal Rules. CRC Press, 2019.

[172] Alexander Tartakovsky, Igor Nikiforov, and Michele Basseville. Sequential analysis: Hypothesis testing and changepoint detection. CRC Press, 2014.

[173] Inder Tecuapetla-Gómez and Axel Munk. Autocovariance estimation in regression with a discontinuous signal and m-dependent errors: A difference-based approach. Scandinavian Journal of Statistics, 44:346-368, 2017.

[174] Robert Tibshirani, Michael Saunders, Saharon Rosset, Ji Zhu, and Keith Knight. Sparsity and smoothness via the fused Lasso. Journal of the Royal Statistical Society, Series B, 67:91-108, 2005.

[175] Ryan J Tibshirani. Adaptive piecewise polynomial estimation via trend filtering. The Annals of Statistics, 42:285-323, 2014.

[176] SO Tickle, IA Eckley, Paul Fearnhead, and Kaylea Haynes. Parallelization of a common changepoint detection method. Journal of Computational and Graphical Statistics, 29:149-161, 2020.

[177] Michalis K Titsias, Christopher C Holmes, and Christopher Yau. Statistical inference in hidden Markov models using k-segment constraints. Journal of the American Statistical Association, 111:200-215, 2016.

[178] Charles Truong, Laurent Oudre, and Nicolas Vayatis. Selective review of offline change point detection methods. Signal Processing, 167:107299, 2020.

[179] E. S. Venkatraman. Consistency Results in Multiple Change-point Problems. PhD thesis, Stanford University, 1992.

[180] ES Venkatraman and Adam B Olshen. A faster circular binary segmentation algorithm for the analysis of array CGH data. Bioinformatics, 23:657-663, 2007.

[181] Daniel Vogel and Martin Wendler. Studentized U-quantile processes under dependence with applications to change-point analysis. Bernoulli, 23(4B):3114-3144, 2017.

[182] L. J. Vostrikova. Detecting 'disorder' in multidimensional random processes. Soviet Doklady Mathematics, 24:55-59, 1981.

[183] Daren Wang, Kevin Lin, and Rebecca Willett. Statistically and computationally efficient change point localization in regression settings. arXiv preprint arXiv:1906.11364, 2019.

[184] Daren Wang, Yi Yu, Alessandro Rinaldo, and Rebecca Willett. Localizing changes in highdimensional vector autoregressive processes. arXiv preprint arXiv:1909.06359, 2019.

[185] Daren Wang, Yi Yu, and Alessandro Rinaldo. Univariate mean change point detection: Penal- 
ization, cusum and optimality. Electronic Journal of Statistics, 14:1917-1961, 2020.

[186] Daren Wang, Yi Yu, and Alessandro Rinaldo. Optimal covariance change point localization in high dimension. Bernoulli, 27:554-575, 2021.

[187] Runmin Wang, Stanislav Volgushev, and Xiaofeng Shao. Inference for change points in high dimensional data. arXiv preprint arXiv:1905.08446, 2019.

[188] Tengyao Wang and Richard J Samworth. High dimensional change point estimation via sparse projection. Journal of the Royal Statistical Society, Series B, 80:57-83, 2018.

[189] Yi-Ching Yao. Estimation of a noisy discrete-time step function: Bayes and empirical Bayes approaches. The Annals of Statistics, pages 1434-1447, 1984.

[190] Yi-Ching Yao. Estimating the number of change-points via Schwarz' criterion. Statistics $\&$ Probability Letters, 6:181-189, 1988.

[191] Yi-Ching Yao. On the asymptotic behavior of a class of nonparametric tests for a change-point problem. Statistics \&3 Probability Letters, 9:173-177, 1990.

[192] Yi-Ching Yao and Siu-Tong Au. Least-squares estimation of a step function. Sankhyā: The Indian Journal of Statistics, Series A, pages 370-381, 1989.

[193] Chun Yip Yau and Zifeng Zhao. Inference for multiple change points in time series via likelihood ratio scan statistics. Journal of the Royal Statistical Society, Series B, 78:895-916, 2016.

[194] Nancy R Zhang and David O Siegmund. A modified Bayes information criterion with applications to the analysis of comparative genomic hybridization data. Biometrics, 63:22-32, 2007.

[195] Zifeng Zhao, Li Chen, and Lizhen Lin. Change-point detection in dynamic networks via graphon estimation. arXiv preprint arXiv:1908.01823, 2019.

[196] Changliang Zou, Guosheng Yin, Long Feng, and Zhaojun Wang. Nonparametric maximum likelihood approach to multiple change-point problems. The Annals of Statistics, 42:970-1002, 2014. 